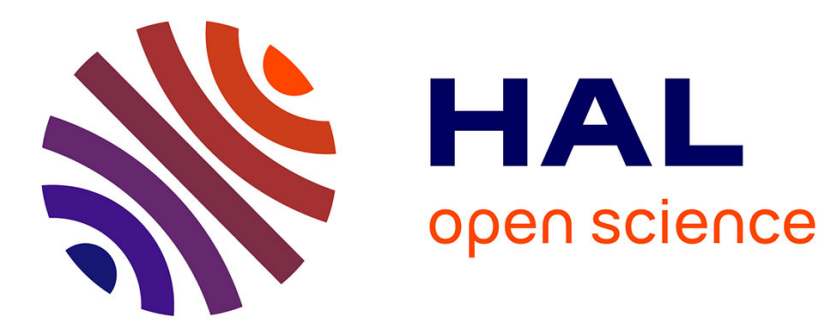

\title{
Noise prevents collapse of Vlasov-Poisson point charges
}

François Delarue, Franco Flandoli, Dario Vincenzi

\section{To cite this version:}

François Delarue, Franco Flandoli, Dario Vincenzi. Noise prevents collapse of Vlasov-Poisson point charges. Communications on Pure and Applied Mathematics, 2014, 67, pp.1700-1736. hal$00683127 \mathrm{v} 2$

\section{HAL Id: hal-00683127 \\ https://hal.science/hal-00683127v2}

Submitted on 5 Jul 2012

HAL is a multi-disciplinary open access archive for the deposit and dissemination of scientific research documents, whether they are published or not. The documents may come from teaching and research institutions in France or abroad, or from public or private research centers.
L'archive ouverte pluridisciplinaire HAL, est destinée au dépôt et à la diffusion de documents scientifiques de niveau recherche, publiés ou non, émanant des établissements d'enseignement et de recherche français ou étrangers, des laboratoires publics ou privés. 


\title{
Noise prevents collapse of Vlasov-Poisson point charges
}

\author{
F. Delarue, F. Flandoli, D. Vincenzi
}

\begin{abstract}
We elucidate the effect of noise on the dynamics of $N$ point charges in a Vlasov-Poisson model driven by a singular bounded interaction force. A too simple noise does not impact the structure inherited from the deterministic case and, in particular, cannot prevent coalescence. Inspired by the theory of random transport in passive scalars, we identify a class of random fields which generate random pulses that are chaotic enough to disorganize the deterministic structure and prevent any collapse of the particles. We obtain strong unique solvability of the stochastic model for any initial configuration of different point charges. In the case where there are exactly two particles, we implement the "vanishing noise method" for determining the continuation of the deterministic model after collapse.
\end{abstract}

\section{Introduction}

It is a well-known fact that white noise perturbations improve the wellposedness properties of Ordinary Differential Equations (ODEs), in particular their uniqueness; see for instance Krylov and Röckner [19]. The influence of noise on pathologies of Partial Differential Equations (PDEs) is not as well understood. A review of recent results in the direction of uniqueness can be found in [12]. On the contrary, whether noise can prevent the emergence of singularities in PDEs is still quite obscure. A further challenging question is whether noise can select a natural candidate for the continuation of solutions after the singularities.

A well-known system in which singularities may develop explicitly is the Vlasov-Poisson equation on the line. We refer the reader to [26] for several 
examples and for an extensive discussion of the underlying stakes, including the connection with the 2D Euler equations. (See also [5], [27] and [34].) The motivation for the present study is to understand the influence of noise on such singularities.

\subsection{Vlasov-Poisson equation on the line}

Consider the following system in the unknown $f:[0, \infty) \times \mathbb{R} \times \mathbb{R} \ni(t, x, v) \mapsto$ $f(t, x, v) \in \mathbb{R}:$

$$
\begin{gathered}
\frac{\partial f}{\partial t}(t, x, v)+v \frac{\partial f}{\partial x}(t, x, v)+E(t, x) \frac{\partial f}{\partial v}(t, x, v)=0, f(0, x, v)=f_{0}(x, v), \\
\rho(t, x)=\int_{\mathbb{R}} f(t, x, v) d v, \quad E(t, x)=\int_{\mathbb{R}} F(x-y) \rho(t, y) d y,
\end{gathered}
$$

where $F(x)$ is a bounded function that is continuous everywhere except at $x=0$ and has side limits in $0^{+}$and $0^{-}$. If $F(x)=\operatorname{sign}(x)($ with $\operatorname{sign}(0)=0)$, Eq. (1) is the 1D Vlasov-Poisson model describing the evolution of the phasespace density $f$ of a system of electrons (in natural units, where the elementary charge and the mass of the electron are set equal to one). Such an equation is known to develop singularities in the case of measure-valued solutions, see [26] and the works discussed therein. For instance, it is possible to design examples of so-called electron-sheet structures that persist in positive time, but collapse to one point at a certain time $\left(f_{0}\right.$ is an electron sheet if it is concentrated on lines, i.e. it reads $\left.f_{0}(x, v)=f_{0}(x) \cdot \delta\left(v-v_{0}(x)\right)\right)$. A simplified version of this phenomenon is the coalescence of $N$ point charges in finite time: as shown below, there are examples of initial conditions of the form $f_{0}(x, v)=$ $\sum_{i=1}^{N} a_{i} \delta\left(x-x_{i}\right) \delta\left(v-v_{i}\right)$, with different pairs $\left(x_{1}, v_{1}\right), \ldots,\left(x_{N}, v_{N}\right) \in \mathbb{R}^{2}$, for which $f$ remains of the same form on some interval $\left[0, t_{0}\right)$ but degenerates into $f\left(t_{0}, x, v\right)=\delta\left(x-x_{0}\right) \delta\left(v-v_{0}\right)$ at some time $t_{0}$, with $\left(x_{0}, v_{0}\right) \in \mathbb{R}^{2}$.

The main question motivating our work is the following: does the presence of noise modify these facts? In this framework, a natural way to conceive a noisy version of (1) may be the following: when the electric charge is not totally isolated, but lives in a medium (a sort of electric bath), a random external force adds to the force generated by the electric field. Assuming that the electric charge does not affect the external random field, the simplest structure modelling this situation is a Stochastic PDE (SPDE) of the form

$$
\frac{\partial f}{\partial t}(t, x, v)+v \frac{\partial f}{\partial x}(t, x, v)+\left(E(t, x)+\varepsilon \circ \frac{d W_{t}}{d t}\right) \frac{\partial f}{\partial v}(t, x, v)=0,
$$


where $W$ is a Brownian motion and the Stratonovich integral is used (this is the natural choice when passing to the limit along regular noises). Unfortunately, the noise in Eq. (2) is too simple to avoid the emergence of singularities such as those described above. Indeed, the random field $\tilde{f}(t, x, v)=f\left(t, x+\varepsilon \int_{0}^{t} W_{s} d s, v+\varepsilon W_{t}\right)$ formally satisfies

$$
\left[\frac{\partial \tilde{f}}{\partial t}+v \frac{\partial \tilde{f}}{\partial x}+\tilde{E} \frac{\partial \tilde{f}}{\partial v}\right](t, x, v)=0 \text { with } \tilde{E}(t, x)=\int_{\mathbb{R}^{2}} F(x-y) \tilde{f}(t, y, v) d y d v
$$

so that any concentration point $z_{0}=\left(x_{0}, v_{0}\right)$ of $\tilde{f}$ at some time $t_{0}$ translates into the random concentration point $\left(x_{0}-\varepsilon \int_{0}^{t_{0}} W_{s} d s, v_{0}-\varepsilon W_{t_{0}}\right)$ of $f$ at the same time.

To expect a positive effect of the noise, we must consider noises of a refined space structure. We consider a noisy equation of the form

$$
\left[d_{t} f+v \frac{\partial f}{\partial x} d t+\left(E(t, x) d t+\varepsilon \sum_{k=1}^{\infty} \sigma_{k}(x) \circ d W_{t}^{k}\right) \frac{\partial f}{\partial v}\right](t, x, v)=0
$$

where $\left(\left(W_{t}^{k}\right)_{t \geq 0}\right)_{k \geq 1}$ is a family of independent Brownian motions, and we prove that, under very general conditions on the covariance function $Q(x, y)=$ $\sum_{k=1}^{\infty} \sigma_{k}(x) \sigma_{k}(y)$, the following result holds:

Theorem 1 Given $f_{0}(x, v)=\sum_{i=1}^{N} a_{i} \delta\left(z-z_{i}\right)$ as initial condition, with the generic notation $z=(x, v)$ and with different initial points $z_{i} \in \mathbb{R}^{2}$, $i=1, \ldots, N$, there is a unique global solution to system (3) of the form $f(t, x, v)=\sum_{i=1}^{N} a_{i} \delta\left(z-z_{i}(t)\right)$, where $\left(\left(z_{i}(t)\right)_{t \geq 0}\right)_{1 \leq i \leq N}$ is a continuous adapted stochastic process with values in $\mathbb{R}^{2 N}$ without coalescence in $\mathbb{R}^{2}$, i.e., with probability one, $z_{i}(t) \neq z_{j}(t)$ for all $t \geq 0$ and $1 \leq i, j \leq N, i \neq j$.

The precise assumptions of Theorem 1 and the definitions used therein will be clarified next. Here, it is worth remarking that our study does not cover the case of an electron sheet. We nonetheless expect our result to be a first step forward in this direction since the number $N$ of particles is here arbitrary for a given covariance function $Q(x, y)$. Indeed, the assumption that we shall impose on $Q(x, y)$ guarantees that any $N$-tuple, with arbitrary $N$, of different points $\left(z_{1}, \ldots, z_{N}\right)$ in the $(x, v)$-space feels the original noise as random impulses that are not too coordinated one with each other. Such a propagation may be seen as a sort of mild spatial chaos produced by the noise. The negative example discussed above does not enjoy a similar property since the noise $\left(\varepsilon W_{t}\right)_{t \geq 0}$ plugged therein produces the same impulse at every space point, thus acting as a random Galilean transformation. 


\subsection{Non-Markovian continuation after a singularity}

As mentioned above, the random perturbation introduced in Eq. (3) may provide some indications about the natural continuation of the solutions after the coalescence of two point charges. (More difficult or generic cases are not clear at this stage of our understanding of the problem.) Consider the simple example in which $F(x)=\operatorname{sign}(x)$ and

$$
f_{0}(x, v)=\frac{1}{2} \delta\left(z-z_{1}\right)+\frac{1}{2} \delta\left(z-z_{2}\right), \quad z_{1}=\left(-1, v_{0}\right), z_{2}=\left(1,-v_{0}\right)
$$

with $v_{0}>0$. As we shall discuss below, the Lagrangian dynamics corresponding to (1) consists of the system

$$
\frac{d x_{i}}{d t}(t)=v_{i}(t), \quad \frac{d v_{i}}{d t}(t)=F\left(x_{i}(t)-x_{\bar{\imath}}(t)\right), \quad t \geq 0,
$$

for $i=1,2$ and $\bar{\imath}=2$ if $i=1$ and vice versa, with $\left(x_{i}(0), v_{i}(0)\right)=\left(\epsilon_{i},-\epsilon_{i} v_{0}\right)$ as initial condition, with $\epsilon_{i}=1$ if $i=2$ and $\epsilon_{i}=-1$ if $i=1$. The functions

$$
v_{i}^{*}(t)=\epsilon_{i}\left(t-v_{0}\right), \quad x_{i}^{*}(t)=\epsilon_{i}\left(1-v_{0} t+\frac{t^{2}}{2}\right)
$$

solve the system for $t \in\left[0, v_{0}\right)$, and the limits of $x_{1}^{*}(t)$ and $x_{2}^{*}(t)$ as $t \uparrow v_{0}$ coincide when $v_{0}^{2}=2$. This means that, with the choice $v_{0}=\sqrt{2}$, the solutions $\left(x_{i}^{*}(t), v_{i}^{*}(t)\right), i=1,2$, converge to the same point $(0,0)$ as $t \uparrow v_{0}$, so that the origin $(0,0)$ is a singular point of the Lagrangian dynamics.

On the contrary, Theorem 1 states that, for any positive level of noise $\varepsilon$ in the noisy formulation (3), the random solutions $\left(\left(x_{i}^{\varepsilon}(t), v_{i}^{\varepsilon}(t)\right)_{t \geq 0}\right)_{i=1,2}$ never meet, with probability one. It is then natural to investigate the behavior of the stochastic solution as $\varepsilon \rightarrow 0$. In Section 2, we shall prove, under general conditions on the covariance function $Q$ :

Theorem 2 Assume that $v_{0}=\sqrt{2}$. Then, as $\varepsilon \rightarrow 0$, the pair process $\left(\left(z_{i}^{\varepsilon}(t)\right)_{t \geq 0}\right)_{i=1,2}$ converges in distribution on the space $\mathcal{C}\left([0, \infty), \mathbb{R}^{2} \times \mathbb{R}^{2}\right)$ toward

$$
\frac{1}{2} \delta\left(\left(z_{1}^{*}(t), z_{2}^{*}(t)\right)_{t \geq 0}\right)+\frac{1}{2} \delta\left(\left(z_{1}^{* *}(t), z_{2}^{* *}(t)\right)_{t \geq 0}\right),
$$

where $\left(z_{i}^{*}(t)=\left(x_{i}^{*}(t), v_{i}^{*}(t)\right)\right.$, for $t \geq 0$ and $i=1,2$, and $\left(z_{1}^{* *}(t), z_{2}^{* *}(t)\right)$ matches $\left(z_{1}^{*}(t), z_{2}^{*}(t)\right)$ for $t \leq \sqrt{2}$ and $\left(z_{2}^{*}(t), z_{1}^{*}(t)\right)$ for $t>\sqrt{2}$. 
Theorem 2 must be seen as a rule for the continuation of the solutions to the deterministic system (1) after singularity. When the particles meet, they split instantaneously, but they can do it in two different ways: $(i)$ with probability $1 / 2$, the trajectories meet at coalescence time and then split without crossing each other, namely each of the two trajectories keeps of constant sign before and after coalescence; $(i i)$ with probability $1 / 2$, the trajectories meet, cross each other and then split for ever, namely the sign of each of them changes at coalescence time exactly. This stands for a mathematical description of the repulsive effect of the interaction force $F$ : there is no way for the particles to merge and then form a single particle with a double charge.

This situation sounds as a physical loss of the Markov property: just after coalescence, splitting occurs because the system keeps memory of what its state was before. Precisely, if we model the dynamics of a static single particle with a double charge by a pair $\left(z_{1}^{00}(t), z_{2}^{00}(t)\right)_{t \geq 0}$ in phase space, subject to $z_{1}^{00}(t)=z_{2}^{00}(t)$ and $\dot{v}_{1}^{00}(t)=\dot{v}_{2}^{00}(t)=0$, for $t \geq 0$, we get a non-Markovian family of solutions to (4). When the trajectories $z_{1}^{0}$ and $z_{2}^{0}$ meet, they do not restart with the same dynamics as $z_{1}^{00}$ and $z_{2}^{00}$ do. We refer the reader to [9] for other mathematical examples of non-Markovian continuations.

\subsection{Vlasov-Poisson type system of $N$ particles in $\mathbb{R}^{d}$}

The problem described in Section 1.1 will be treated as a particular case of the following generalization in $\mathbb{R}^{d}$ :

$$
\left[d_{t} f+\left(v \cdot \nabla_{x} f+E(t, x) \cdot \nabla_{v} f\right) d t+\sum_{k=1}^{\infty} \sigma_{k}(x) \cdot \nabla_{v} f \circ d W_{t}^{k}\right](t, x, v)=0
$$

subject to similar constraints as in (1), where $\sigma_{k}: \mathbb{R}^{d} \rightarrow \mathbb{R}^{d}$ are Lipschitzcontinuous fields subject to additional assumptions, which will be specified later (see (A.2-3) in Section 3), and $\left(\left(W_{t}^{k}\right)_{t \geq 0}\right)_{k \in \mathbb{N} \backslash\{0\}}$ are independent onedimensional Brownian motions. Below, $F$ will be assumed to be bounded and locally Lipschitz continuous on any compact subset of $\mathbb{R}^{d} \backslash\{0\}$, the Lipschitz constant on any ring of the form $\left\{x \in \mathbb{R}^{d}: r \leq|x| \leq 1\right\}$ growing at most as $1 / r$ as $r$ tends to 0 . In particular, $F$ may be discontinuous at 0 . A relevant case is when $F=\nabla U$ where $U$ is a potential with a Lipschitz point at zero, namely $U$ is Lipschitz continuous on $\mathbb{R}^{d}$ and smooth on $\mathbb{R}^{d} \backslash\{0\}$.

This framework includes the example $F(x)=x /|x|, x \in \mathbb{R}^{d}$, and, as a particular case, the one-dimensional model discussed above, i.e. $F(x)=$ 
$\operatorname{sign}(x), x \in \mathbb{R}$. On the contrary, the $d$-dimensional Poisson case, where

$$
F(x)= \pm x|x|^{-d}, \quad x \in \mathbb{R}^{d}, \quad d \geq 2,
$$

does not satisfy the aforementioned assumptions, and therefore falls outside this study. The signs "+" and "-" describe repulsive and attractive interactions respectively; corresponding models are referred to as electrostatic and gravitational respectively. For the electrostatic potential, our analysis turns out to be irrelevant in dimension $d \geq 2$ since the deterministic system itself is free of coalescence.

The Lagrangian motion associated with the SPDE is

$$
\frac{d X_{t}^{i}}{d t}=V_{t}^{i}, \quad d V_{t}^{i}=\sum_{j \neq i} a_{j} F\left(X_{t}^{i}-X_{t}^{j}\right) d t+\sum_{k=1}^{\infty} \sigma_{k}\left(X_{t}^{i}\right) \circ d W_{t}^{k},
$$

for $t \geq 0$ and $1 \leq i \leq N$. The measure valued process

$$
f(t, x, v)=\sum_{i=1}^{N} a_{i} \delta\left(x-X_{t}^{i}\right) \delta\left(v-V_{t}^{i}\right)
$$

solves the SPDE in weak form, from Itô's formula in Stratonovich form applied to $\left(\sum_{1 \leq i \leq N} a_{i} \varphi\left(X_{t}^{i}, V_{t}^{i}\right)\right)_{t \geq 0}$, for a test function $\varphi: \mathbb{R}^{d} \times \mathbb{R}^{d} \rightarrow \mathbb{R}$. This paper is devoted to the analysis of system (8). Taking this system as a starting point, the problem would be much easier to handle if each particle were to be forced with its own Brownian motion, independently of the other ones. This choice of the noise, however, would break the relation between the Lagrangian dynamics and the SPDE introduced above.

The paper is organized as follows. In Section 2, we start with the proof of Theorem 2, as we feel it to have a striking interpretation from the physical point of view. The vanishing noise method for selecting solutions to singular differential equations goes back to the earlier paper by [3], but the examples investigated therein are one-dimensional only. (See also [12], together with [15] and [2].) Here Theorem 2 applies to a four-dimensional system. In [3], the vanishing noise behavior of the stochastic differential equation at hand is mainly of analytical essence. Below, the proof of Theorem 2 relies on a new approach: pathwise, the asymptotic dynamics of $\left(z_{i}^{\varepsilon}\right)_{i=1,2}$ are expanded with respect to the parameter $\varepsilon$ till coalescence occurs; the possible limit regimes then read on the (random) coefficients of the expansion. The result 
is obtained under general conditions on the covariance matrix $Q$ : specific examples are given in Section 3.

The remainder of the paper is devoted to the proof of Theorem 1 . We first discuss the structure of the noise that will prevent coalescence from emerging. It should be emphasized that the effect of the noise on the $2 \mathrm{~N}$ system (8) is highly non-trivial. Indeed, although it is doubly singular, the noise makes the system fluctuate enough to avoid pathological phenomena such as those observed in the deterministic case. The first singularity is due to the fact that the same Brownian motions act on all the particles. If the $\left(\sigma_{k}\right)_{k \in \mathbb{N} \backslash\{0\}}$ were constant, the particles would feel the same impulses and the noise would not have any real effect; basically, it would just act as a random translation of the system, as in (2). Thus the point is to design a noise allowing displacements of different particles in different directions. To reach the desired effect, we require the covariance matrix $\left(Q\left(x^{i}, x^{j}\right)\right)_{1 \leq i, j \leq N}$ to be strictly positive for any vector $\left(x^{1}, \ldots, x^{N}\right) \in\left(\mathbb{R}^{d}\right)^{N}$ with pairwise different entries. We give examples in Section 3. These examples are inspired from the Kraichnan noise used in the theory of random transport of passive scalars. (See for example [11], [22] and [29].) However, the model considered here does not have the same interpretation as the Kraichnan model, since the noise here acts onto the velocities. A possible way to relate Eq. (8) to turbulence theory would consist in penalizing the drift of the velocity of the $i$ th particle by $-V_{t}^{i}$. This model would describe the motion of interacting heavy particles in a fluid whose velocity is a random field, see [4].

The second singularity of the model is inherited from the kinetic structure of the deterministic counterpart: the noise acts as an additional random force only, namely it is plugged into the equation of the velocity only. In other words, the coupled system for $\left(X_{t}^{i}, V_{t}^{i}\right)_{1 \leq i \leq N}$ is degenerate. We shall show in Subsection 3.3 that the ellipticity properties of the noise in $\mathbb{R}^{N d}$ actually lift up to hypoellipticity properties in $\mathbb{R}^{2 N d}$.

Once the set-up for the noise is defined, we are ready to tackle the problem of no-coalescence. We first establish that the Lagrangian dynamics are well posed for Lebesgue a.e. initial configuration of different particles. This does not require any special features of the noise. Specifying the form of the noise according to the requirements discussed in Section 3, we then prove well-posedness and no-collapse for all initial conditions of the particle system with pairwise different entries by taking advantage of the hypoellipticity property of the whole system, see Subsection 4.3. The main lines of Section 4 are connected with the strategy already developed by [13] in order to prove 
that noise may prevent $N$ point vertices, when driven by $2 \mathrm{D}$ Euler equations, from collapsing. However, both the framework and the results are here quite different. In [13], the noise is finite dimensional, the dimension depending upon the number of particles; it is given implicitly only, from a generic existence result; moreover, the dynamics of the particles are non-degenerate. Here, the structure of the noise is explicit and is independent of the number of particles; morever, the dynamics of the particles are degenerate.

\subsection{Assumption}

For simplicity, we choose $a_{i}=1 / N$ for $1 \leq i \leq N$. We also assume that

(A.1) $F$ is bounded everywhere on $\mathbb{R}^{d}$ and locally Lipschitz-continuous on any compact subset of $\mathbb{R}^{d} \backslash\{0\}$. Moreover,

$$
\sup _{0<r \leq 1} \sup _{r \leq|x|,|y| \leq 1, x \neq y}\left[r \frac{|F(x)-F(y)|}{|x-y|}\right]<+\infty .
$$

The examples we have in mind are: for $d=1, F(x)=\operatorname{sgn}(x), x \in \mathbb{R} \backslash\{0\}$; for $d \geq 2, F(x)=x /|x|, x \in \mathbb{R}^{d} \backslash\{0\}$.

(A.2) For each $k \in \mathbb{N} \backslash\{0\}, \sigma_{k}: \mathbb{R}^{d} \rightarrow \mathbb{R}^{d}$ is Lipschitz-continuous, and the series $\sum_{k=1}^{\infty} \sigma_{k}^{\alpha}(\tilde{x}) \sigma_{k}^{\beta}(\tilde{y})$ converges uniformly w.r.t. $(\tilde{x}, \tilde{y})$ in compact subset of $\mathbb{R}^{d} \times \mathbb{R}^{d}$, for each $\alpha, \beta=1, \ldots, d$. We define

$$
Q(\tilde{x}, \tilde{y})=\sum_{k=1}^{\infty} \sigma_{k}(\tilde{x}) \otimes \sigma_{k}(\tilde{y}) \in \mathbb{R}^{d \times d}
$$

as the covariance function of the random field $\mathbb{R}^{d} \ni \tilde{x} \mapsto \sum_{k=1}^{\infty} \sigma_{k}(\tilde{x}) W_{1}^{k}$. It is of positive type, that is $\sum_{i, j=1}^{n}\left\langle Q\left(\tilde{x}^{j}, \tilde{x}^{i}\right) \tilde{v}^{i}, \tilde{v}^{j}\right\rangle_{\mathbb{R}^{d}} \geq 0$, for any $n \geq 1$, $\tilde{x}^{1}, \ldots, \tilde{x}^{n}, \tilde{v}^{1}, \ldots, \tilde{v}^{n} \in \mathbb{R}^{d}$.

(A.3) $Q(\tilde{x}, \tilde{y})$ is bounded on the diagonal, that is $\sup _{\tilde{x} \in \mathbb{R}^{d}}|Q(\tilde{x}, \tilde{x})|<$ $+\infty$. Moreover, it satisfies the Lipschitz type regularity property

$$
\sup _{\tilde{x}, \tilde{y} \in \mathbb{R}^{d} \tilde{x} \neq \tilde{y}} \frac{|Q(\tilde{x}, \tilde{x})+Q(\tilde{y}, \tilde{y})-Q(\tilde{x}, \tilde{y})-Q(\tilde{y}, \tilde{x})|}{|\tilde{x}-\tilde{y}|^{2}}<+\infty .
$$

(A.4) $Q(\tilde{x}, \tilde{y})$ is strictly positive on $\Gamma_{x, N}=\left\{\left(x^{1}, \ldots, x^{N}\right) \in \mathbb{R}^{N d}\right.$ : $x^{i} \neq x^{j}$ whenever $\left.i \neq j\right\}$, that is, for all $\left(x^{1}, \ldots, x^{N}\right) \in \Gamma_{x, N}$ and $v=$ $\left(v^{1}, \ldots, v^{N}\right) \in \mathbb{R}^{N d} \backslash\{0\}, \sum_{i, j=1}^{N}\left\langle Q\left(x^{j}, x^{i}\right) v^{i}, v^{j}\right\rangle_{\mathbb{R}^{d}}>0$. 
The regularity assumptions on $\sigma_{k}$ and $Q$ in (A.2) and (A.3) respectively are strongly related one with each other. Specifically, the Lipschitz condition (11) implies a strong Lipschitz property of the fields $\left(\sigma_{k}\right)_{k \in \mathbb{N} \backslash\{0\}}$ :

$$
\begin{aligned}
& \sum_{k=1}^{\infty}\left(\sigma_{k}^{\alpha}(\tilde{x})-\sigma_{k}^{\alpha}(\tilde{y})\right)\left(\sigma_{k}^{\beta}(\tilde{x})-\sigma_{k}^{\beta}(\tilde{y})\right) \\
& =Q_{\alpha \beta}(\tilde{x}, \tilde{x})-Q_{\alpha \beta}(\tilde{x}, \tilde{y})-Q_{\alpha \beta}(\tilde{y}, \tilde{x})+Q_{\alpha \beta}(\tilde{y}, \tilde{y}) \leq C|\tilde{x}-\tilde{y}|^{2} .
\end{aligned}
$$

Conversely, Eq. (11) holds if the Lipschitz constants of the $\left(\sigma_{k}\right)_{k \in \mathbb{N} \backslash\{0\}}$ are square summable.

In practice, the covariance function $Q$ is given first. Precisely, given a function $Q: \mathbb{R}^{2 d} \ni(\tilde{x}, \tilde{y}) \mapsto Q(\tilde{x}, \tilde{y})$ with values in the set of symmetric matrices of size $d \times d$, satisfying (A.3) and of positive type, it may be expressed as a covariance function as in (10), for some fields $\left(\sigma_{k}\right)_{k \in \mathbb{N} \backslash\{0\}}$ satisfying (A.2). We refer to Theorem 4.2.5 in [20]. In this framework, a sufficient condition to guarantee (11) is: $Q$ is of class $\mathcal{C}^{2}$ with bounded mixed derivatives, that is $\sup _{(\tilde{x}, \tilde{y}) \in \mathbb{R}^{d} \times \mathbb{R}^{d}}\left|\partial_{\tilde{x}, \tilde{y}}^{2} Q(\tilde{x}, \tilde{y})\right|<+\infty$. Indeed, Lipschitz property (11) then follows from a straightforward Taylor expansion.

By (A.2), Stratonovich integrals in SDE (8) are (formally) equal to Itô integrals, so that (8) will be interpreted in the usual Itô form

$$
\frac{d X_{t}^{i}}{d t}=V_{t}^{i}, \quad d V_{t}^{i}=\frac{1}{N} \sum_{j \neq i} F\left(X_{t}^{i}-X_{t}^{j}\right) d t+\sum_{k=1}^{\infty} \sigma_{k}\left(X_{t}^{i}\right) d W_{t}^{k},
$$

$t \geq 0, i \in\{1, \ldots, N\}$. Indeed, the local martingale part of $\left(\sigma_{k}\left(X_{t}^{i}\right)\right)_{t \geq 0}$ is zero, since $\left(\sigma_{k}\left(X_{t}^{i}\right)\right)_{t \geq 0}$ is of bounded variation. We shall not treat more rigorously this equivalence, and, from now, we shall adopt the Itô formulation.

\subsection{Useful notation}

In the whole paper, the number $N$ of particles is fixed, so that the dependence of constants upon $N$ is not investigated. For any $n \in \mathbb{N} \backslash\{0\}, z \in \mathbb{R}^{n}$ and $r>0, B_{n}(z, r)$ is the closed ball of dimension $n$, center $z$ and radius $r$; Leb ${ }_{n}$ is the Lebesgue measure on $\mathbb{R}^{n}$. The volume of $B_{n}(z, r)$ is denoted by $\mathcal{V}_{n}(r)$. The configurations of the $N$-particle system in the phase space are generally denoted by $z$ or $Z$. Positions are denoted by $x$ or $X$ and velocities by $v$ or $V$. Similarly, the typical notation for a single particle in the phase space is $\tilde{z}=(\tilde{x}, \tilde{v}), \tilde{x}$ standing for its position and $\tilde{v}$ for its velocity. The set of 
pairs of different indices in the particle system is denoted by $\Delta_{N}=\{(i, j) \in$ $\left.\{1, \ldots, N\}^{2}: i \neq j\right\}$. Moreover, we set $\Gamma_{N}=\left\{\left(z^{1}, \ldots, z^{N}\right) \in \mathbb{R}^{2 N d}: \forall(i, j) \in\right.$ $\left.\Delta_{N}, z_{i} \neq z_{j}\right\}$ and $\Gamma_{x, N}=\left\{\left(x^{1}, \ldots, x^{N}\right) \in \mathbb{R}^{N d}: \forall(i, j) \in \Delta_{N}, x_{i} \neq x_{j}\right\}$. We also define the projection mappings:

$$
\begin{aligned}
& \Pi_{x}: \mathbb{R}^{2 N d} \ni z=\left(z^{j}\right)_{1 \leq j \leq N}=\left(\left(x^{j}, v^{j}\right)\right)_{1 \leq j \leq N} \mapsto \Pi_{x}(z)=\left(x^{j}\right)_{1 \leq j \leq N} \in \mathbb{R}^{N d}, \\
& \tilde{\pi}_{x}: \mathbb{R}^{2 d} \ni \tilde{z}=(\tilde{x}, \tilde{v}) \mapsto \tilde{x} \in \mathbb{R}^{d}, \\
& \pi_{i, x}: \mathbb{R}^{2 N d} \ni z=\left(z^{j}\right)_{1 \leq j \leq N}=\left(\left(x^{j}, v^{j}\right)\right)_{1 \leq j \leq N} \mapsto \pi_{i, x}(z)=x^{i} \in \mathbb{R}^{d},
\end{aligned}
$$

with a similar definition for $\Pi_{v}, \tilde{\pi}_{v}$ and $\pi_{i, v}$. We then put $\pi_{i}=\left(\pi_{i, x}, \pi_{i, v}\right)$.

Below, Eq. (13) will be also written in the compact form

$$
d Z_{t}=\mathbb{F}\left(Z_{t}\right) d t+\sum_{k=1}^{\infty} \mathbb{A}_{k}\left(Z_{t}\right) d W_{t}^{k}, \quad t \geq 0
$$

where $Z_{t}=\left(X_{t}, V_{t}\right)$, with $X_{t}=\left(X_{t}^{1}, \ldots, X_{t}^{N}\right)$ and $V_{t}=\left(V_{t}^{1}, \ldots, V_{t}^{N}\right)$, and

$$
\begin{aligned}
& \mathbb{F}: \mathbb{R}^{N d} \times \mathbb{R}^{N d} \ni\left(x=\left(x^{1}, \ldots, x^{N}\right), v\right) \mapsto\left(v,\left(\frac{1}{N} \sum_{j \neq i} F\left(x^{i}-x^{j}\right)\right)_{1 \leq i \leq N}\right) \in \mathbb{R}^{N d} \times \mathbb{R}^{N d}, \\
& \mathbb{A}_{k}: \mathbb{R}^{N d} \times \mathbb{R}^{N d} \ni(x, v) \mapsto\left(0, A_{k}(x)\right) \in \mathbb{R}^{N d} \times \mathbb{R}^{N d}, \\
& A_{k}:\left(\mathbb{R}^{d}\right)^{N} \ni\left(x^{1}, \ldots, x^{N}\right) \mapsto\left(\sigma_{k}\left(x^{1}\right), \ldots, \sigma_{k}\left(x^{N}\right)\right) \in\left(\mathbb{R}^{d}\right)^{N} .
\end{aligned}
$$

For any $t \geq 0$, the $2 d$-coordinates of $Z_{t}$ will be denoted by $Z_{t}^{i}=\pi_{i}\left(Z_{t}\right)=$ $\left(X_{t}^{i}, V_{t}^{i}\right), i \in\{1, \ldots, N\}$. Similarly, we shall denote by $\left(\mathbb{F}^{i} \equiv \pi_{i}(\mathbb{F})\right)_{1 \leq i \leq N}$ and $\left(\mathbb{A}_{k}^{i} \equiv \pi_{i}\left(\mathbb{A}_{k}\right)\right)_{1 \leq i \leq N}$ the $2 d$-components of $\mathbb{F}$ and $\mathbb{A}_{k}$, for $k \in \mathbb{N} \backslash\{0\}$.

\section{Continuation: Proof of Theorem 2}

Here, we identify general conditions on the structure of the noise in (3) under which Theorem 2 holds. Typical examples are given in Proposition 19 in Section 3. In the whole section, we thus consider the 4D system:

$$
d X_{t}^{i, \varepsilon}=V_{t}^{i, \varepsilon} d t, \quad d V_{t}^{i, \varepsilon}=\operatorname{sign}\left(X_{t}^{i, \varepsilon}-X_{t}^{\bar{i}, \varepsilon}\right)+\varepsilon \sum_{k=1}^{\infty} \sigma_{k}\left(X_{t}^{i, \varepsilon}\right) d W_{t}^{k},
$$

for $t \geq 0, i=1,2$ and $\bar{l}=2$ if $i=1$ and vice versa. Below, we assume that $\left(X_{0}^{i, \varepsilon}, V_{0}^{i, \varepsilon}\right)=\left(\epsilon_{i},-\epsilon_{i} \sqrt{2}\right)$, with $\epsilon_{i}=1$ if $i=2$ and $\epsilon_{i}=-1$ if $i=1$. As a first general condition (again, we refer to Proposition 19 for examples), we set: 
Condition 3 For any $\varepsilon>0$, Theorem 1 applies and thus (16) has a unique strong solution, which satisfies $\mathbb{P}\left\{\forall t \geq 0,\left(X_{t}^{1, \varepsilon}, V_{t}^{1, \varepsilon}\right) \neq\left(X_{t}^{2, \varepsilon}, V_{t}^{2, \varepsilon}\right)\right\}=1$.

We will set $Z_{t}^{i, \varepsilon}=\left(X_{t}^{i, \varepsilon}, V_{t}^{i, \varepsilon}\right), \varepsilon>0, i=1,2$. When $\varepsilon=0$, the curves

$$
X_{t}^{i, 0}=\epsilon_{i}\left(1-\frac{t}{\sqrt{2}}\right)^{2}, \quad V_{t}^{i, 0}=\epsilon_{i}(-\sqrt{2}+t), \quad t \geq 0
$$

solve the system (16), but merge at time $t_{0}=\sqrt{2}$. Again, we will set $Z_{t}^{i, 0}=$ $\left(X_{t}^{i, 0}, V_{t}^{i, 0}\right), i=1,2$. Note that $Z_{t}^{2,0}=-Z_{t}^{1,0}$ for all $t \geq 0$.

We are to prove that $\left(Z_{t}^{1, \varepsilon}, Z_{t}^{2, \varepsilon}\right)_{t \geq 0}$ converges in distribution on the space $\mathcal{C}\left([0,+\infty), \mathbb{R}^{4}\right)$ toward $(1 / 2) \delta_{\left(Z_{t}^{1,+}, Z_{t}^{2,+}\right)_{t \geq 0}}+(1 / 2) \delta_{\left(Z_{t}^{1,-}, Z_{t}^{2,-}\right)_{t \geq 0}}$, where

$$
\left(Z_{t}^{1,+}, Z_{t}^{2,+}\right)=\left(Z_{t}^{1,0}, Z_{t}^{2,0}\right), t \geq 0 ; \quad\left(Z_{t}^{1,-}, Z_{t}^{2,-}\right)=\left\{\begin{array}{l}
\left(Z_{t}^{1,0}, Z_{t}^{2,0}\right), t \in\left[0, t_{0}\right] \\
\left(Z_{t}^{2,0}, Z_{t}^{1,0}\right), t>t_{0} .
\end{array}\right.
$$

The whole point is to investigate the differences:

$$
\mathbb{X}_{t}^{\varepsilon}=\frac{X_{t}^{2, \varepsilon}-X_{t}^{1, \varepsilon}}{2}, \quad \mathbb{V}_{t}^{\varepsilon}=\frac{V_{t}^{2, \varepsilon}-V_{t}^{1, \varepsilon}}{2}, \quad t \geq 0, \quad \varepsilon>0 .
$$

We will use the second condition (see as an example Proposition 19):

Condition 4 Assume that $\left(Z_{t}^{1, \varepsilon}, Z_{t}^{2, \varepsilon}\right)_{t \geq 0}$ satisfies (16), but with an arbitrary random initial condition $\left(Z_{0}^{1, \varepsilon}, Z_{0}^{2, \varepsilon}\right) \in \Gamma_{2}$, independent of the noise $\left(\left(W_{t}^{k}\right)_{t \geq 0}\right)_{k \geq 1}$. Denote by $\left(\mathcal{F}_{t}\right)_{t \geq 0}$ the augmented filtration generated by the initial condition $\left(Z_{0}^{1, \varepsilon}, Z_{0}^{2, \varepsilon}\right)$ and by the noise $\left(\left(W_{t}^{k}\right)_{t \geq 0}\right)_{k \geq 1}$. Then, there exists an $\left(\mathcal{F}_{t}\right)_{t \geq 0}$-Brownian motion $\left(B_{t}^{\varepsilon}\right)_{t \geq 0}$ such that, for all $t \geq 0$,

$$
d \mathbb{X}_{t}^{\varepsilon}=\mathbb{V}_{t}^{\varepsilon} d t, \quad d \mathbb{V}_{t}^{\varepsilon}=\operatorname{sign}\left(\mathbb{X}_{t}^{\varepsilon}\right) d t+\varepsilon \sigma\left(\mathbb{X}_{t}^{\varepsilon}\right) d B_{t}^{\varepsilon},
$$

where $\sigma$ is $\mathcal{C}^{2}$ function from $\mathbb{R}$ to $\mathbb{R}$, depending on the $\left(\sigma_{k}\right)_{k \geq 1}$ only (in particular, $\sigma$ is independent of the initial condition $\left(Z_{0}^{1, \varepsilon}, Z_{0}^{2, \varepsilon}\right)$ and of $\varepsilon$ ), with bounded derivatives of order 1 and 2 , such that $\sigma(0)=0$ and $\sigma(1)>0$.

Setting $\mathbb{Z}_{t}^{\varepsilon}=\left(\mathbb{X}_{t}^{\varepsilon}, \mathbb{V}_{t}^{\varepsilon}\right)$, for any $t \geq 0$, we first investigate the solutions of (18) when $\varepsilon=0$. We have the obvious

Lemma 5 For $\varepsilon=0$, all the solutions of (18) with $\varepsilon=0$ and $\left(\mathbb{X}_{0}^{0}, \mathbb{V}_{0}^{0}\right)=$ $(1,-\sqrt{2})$, have the form

$$
\mathbb{Z}_{t}^{0}=\left(\mathbb{X}_{t}^{0}, \mathbb{V}_{t}^{0}\right)=\left(\frac{\left(t_{0}-t\right)^{2}}{2}, t-t_{0}\right) \quad \text { for } \quad 0 \leq t \leq t_{0}=\sqrt{2}
$$


We emphasize that uniqueness fails after coalescence time $t_{0}$. Indeed, any $\left(\mathbb{Z}_{t}^{0}\right)_{t \geq 0}$, with $\left(\mathbb{Z}_{t}^{0}\right)_{0 \leq t \leq t_{0}}$ as in $(19), \mathbb{Z}_{t}^{0}=(0,0)$ for $t_{0} \leq t \leq t_{1}$ and $\mathbb{Z}_{t}^{0}=$ $\pm\left(\left(t-t_{1}\right)^{2} / 2, t-t_{1}\right)$, for $t \geq t_{1}$, where $t_{1} \geq t_{0}$ may be real or infinite, is a solution to (18) when $\varepsilon=0$ therein. We claim

Proposition 6 Given $\tau^{\varepsilon}=\inf \left\{t \geq 0: \mathbb{X}_{t}^{\varepsilon} \leq 0\right\}$, for any $\delta>0$ and $M>t_{0}$,

$$
\lim _{\varepsilon \rightarrow 0} \mathbb{P}\left\{\tau^{\varepsilon} \in\left(t_{0}-\delta, t_{0}+\delta\right)\right\}=\frac{1}{2}, \quad \lim _{\varepsilon \rightarrow 0} \mathbb{P}\left\{\tau^{\varepsilon} \geq M\right\}=\frac{1}{2} .
$$

Moreover, defining $\tau_{2}^{\varepsilon}=\inf \left\{t>\tau^{\varepsilon}: \mathbb{X}_{t}^{\varepsilon} \geq 0\right\}$, we have, for any $M>0$,

$$
\lim _{\varepsilon \rightarrow 0} \mathbb{P}\left\{\tau_{2}^{\varepsilon} \geq M\right\}=1 \text {. }
$$

Proposition 6 suggests that, in the limit regime $\varepsilon \rightarrow 0$, the trajectories of the two particles cross with probability $1 / 2$ exactly, and, if so, they just cross once, at coalescence time. This is one step forward in the proof of Theorem 2. Precisely, we prove below that Proposition 6 implies Theorem 2.

Proof. (Proposition $6 \Rightarrow$ Theorem 2.) The family $\left(\left(Z_{t}^{1, \varepsilon}, Z_{t}^{2, \varepsilon}\right)_{t \geq 0}\right)_{0<\varepsilon \leq 1}$ is tight by (A.3). We denote by $\mu$ a weak limit, on the space of continuous functions $\mathcal{C}\left([0,+\infty), \mathbb{R}^{4}\right)$, of the family of measures $\left(\mathbb{P}_{\left(Z^{1, \varepsilon}, Z^{2, \varepsilon}\right)}\right)_{0<\varepsilon \leq 1}$ as $\varepsilon \rightarrow 0$, the canonical process on $\mathcal{C}\left([0,+\infty), \mathbb{R}^{4}\right)$ being denoted by $\left(\xi_{.}^{1}=\left(\chi_{.}^{1}, \nu_{.}^{1}\right), \xi^{2}=\right.$ $\left.\left(\chi_{.}^{2}, \nu_{.}^{2}\right)\right)$. We will also denote $\chi_{t}=\left(\chi_{t}^{2}-\chi_{t}^{1}\right) / 2$ and $\nu_{t}=\left(\nu_{t}^{2}-\nu_{t}^{1}\right) / 2, t \geq 0$.

Under the measure $\mu, \xi^{i}=\left(\chi_{.}^{i}, \nu_{.}^{i}\right), i=1,2$, satisfies $\dot{\chi}_{t}^{i}=\nu_{t}^{i},\left|\dot{\nu}_{t}^{i}\right| \leq 1$, $t \geq 0, i=1,2$. We now make use of Proposition 6. Given $M>0$, we have, on the set $\left\{\tau^{\varepsilon} \geq M\right\}$,

$$
V_{t}^{i, \varepsilon}=V_{0}^{i, \varepsilon}+\epsilon_{i} t+\varepsilon \sum_{k \geq 1} \int_{0}^{t} \sigma_{k}\left(X_{s}^{i, \varepsilon}\right) d W_{s}^{k}, \quad 0 \leq t \leq M,
$$

where $\epsilon_{i}$ matches 1 if $i=2$ and -1 if $i=1$, so that, for any $\eta>0$,

$$
\liminf _{\varepsilon \rightarrow 0} \mathbb{P}\left\{\sup _{0 \leq t \leq M}\left|V_{t}^{i, \varepsilon}-V_{0}^{i, \varepsilon}-\epsilon_{i} t\right| \leq \eta, \quad i=1,2\right\} \geq \lim _{\varepsilon \rightarrow 0} \mathbb{P}\left\{\tau^{\varepsilon} \geq M\right\} .
$$

By porte-manteau Theorem, we deduce that

$$
\mu\left\{\nu_{t}^{i}=\nu_{0}^{i}+\epsilon_{i} t, 0 \leq t \leq M, i=1,2\right\} \begin{cases}=1 & \text { if } M<t_{0}, \\ \geq 1 / 2 & \text { if } M>t_{0} .\end{cases}
$$


Therefore, under $\mu,\left(\xi_{t}^{1}\right)_{0 \leq t \leq t_{0}}$ coincides with $\left(Z_{t}^{1,0}\right)_{0 \leq t \leq t_{0}}$ and $\left(\xi_{t}^{2}\right)_{0 \leq t \leq t_{0}}$ coincides with $\left(Z_{t}^{2,0}\right)_{0 \leq t \leq t_{0}}$. In particular, under $\mu, \xi_{t_{0}}^{1}=\xi_{t_{0}}^{2}=(0,0)$. Similarly, we also deduce from (22) that, with probability greater than $1 / 2$ under $\mu$, $\left(\xi_{t}^{1}, \xi_{t}^{2}\right)=\left(Z_{t}^{1,+}, Z_{t}^{2,+}\right)$ for any $t \geq 0$.

By the same argument, for $\delta>0$ small and $M>t_{0}+\delta$, we deduce from Proposition 6 that,

$$
\begin{aligned}
& \mu\left\{\nu_{t}^{i}=\nu_{t_{0}+\delta}^{i}-\epsilon_{i}\left[t-\left(t_{0}+\delta\right)\right], \quad t_{0}+\delta \leq t \leq M, \quad i=1,2\right\} \\
& \geq \lim _{\varepsilon \rightarrow 0} \mathbb{P}\left\{\tau^{\varepsilon} \leq t_{0}+\delta, \tau_{2}^{\varepsilon} \geq M\right\}=\frac{1}{2} .
\end{aligned}
$$

Letting $\delta$ tend toward 0 and $M$ toward $+\infty$, we deduce that, with probability greater than $1 / 2$ under $\mu,\left(\xi_{t}^{1}, \xi_{t}^{2}\right)=\left(Z_{t}^{1,-}, Z_{t}^{2,-}\right)$ for any $t \geq 0$.

\subsection{Key Lemmas by Integration by Parts}

The proof of Proposition 6 relies on two key lemmas, each of them being proven by integration by parts. The first one is

Lemma 7 Set $N^{+}=[0,+\infty)^{2} \backslash\{(0,0)\}$ and, similarly, $N^{-}=(-\infty, 0]^{2} \backslash$ $\{(0,0)\}$. Consider also the sets of initial conditions for $(16): \Gamma^{ \pm}=\left\{\left(z^{1}=\right.\right.$ $\left.\left.\left(x^{1}, v^{1}\right), z^{2}=\left(x^{2}, v^{2}\right)\right):\left(x^{2}-x^{1}, v^{2}-v^{1}\right) \in N^{ \pm}\right\}$. Then, there exists a constant $c>0$ such that, for any $M>0$ and any compact subset $K \subset \mathbb{R}^{4}$,

$$
\lim _{\varepsilon \rightarrow 0} \inf _{\left(z^{1}, z^{2}\right) \in K \cap \Gamma^{ \pm}} \mathbb{P}\left\{\forall t \in[0, M], \pm \mathbb{X}_{t}^{\varepsilon} \geq c t^{2} \mid\left(Z_{0}^{1, \varepsilon}, Z_{0}^{2, \varepsilon}\right)=\left(z^{1}, z^{2}\right)\right\}=1 .
$$

Proof. In the whole proof, the initial condition $\left(z^{1}, z^{2}\right) \in K \cap \Gamma^{+}$is given, i.e. $\left(Z_{0}^{1, \varepsilon}, Z_{0}^{2, \varepsilon}\right)=\left(z^{1}, z^{2}\right) \in K \cap \Gamma^{+}$. Writing $z^{i}=\left(x^{i}, v^{i}\right), i=1$, 2 , we set $x=\left(x^{2}-x^{1}\right) / 2$ and $v=\left(v^{2}-v^{1}\right) / 2$. W.l.o.g., we assume that $x>0$. Indeed, when $x=0, v$ must be positive, so that, in very short time, both $\mathbb{X}^{\varepsilon}$ and $\mathbb{V}^{\varepsilon}$ are positive. By Markov property (which holds for the 4D system because of strong uniqueness), we are then led back to the case when $x$ and $v$ are positive. By Condition 4, we can write

$$
d \mathbb{V}_{t}^{\varepsilon}=\operatorname{sign}\left(\mathbb{X}_{t}^{\varepsilon}\right) d t+\varepsilon \sigma\left(\mathbb{X}_{t}^{\varepsilon}\right) d B_{t}^{\varepsilon}, \quad t \geq 0,
$$

where $\left(B_{t}^{\varepsilon}\right)_{t \geq 0}$ is a $1 \mathrm{D}$ Browian motion. Taking advantage of the smoothness of $\sigma$, we perform the following integration by parts:

$$
d\left(\mathbb{V}_{t}^{\varepsilon}-\varepsilon \sigma\left(\mathbb{X}_{t}^{\varepsilon}\right) B_{t}^{\varepsilon}\right)=\left(\operatorname{sign}\left(\mathbb{X}_{t}^{\varepsilon}\right)-\varepsilon \sigma^{\prime}\left(\mathbb{X}_{t}^{\varepsilon}\right) \mathbb{V}_{t}^{\varepsilon} B_{t}^{\varepsilon}\right) d t
$$


Keeping in mind that $\tau^{\varepsilon}=\inf \left\{t \geq 0: \mathbb{X}_{t}^{\varepsilon} \leq 0\right\}$, we have

$$
\mathbb{V}_{t}^{\varepsilon}-\varepsilon \sigma\left(\mathbb{X}_{t}^{\varepsilon}\right) B_{t}^{\varepsilon} \geq t-\varepsilon \int_{0}^{t} \sigma^{\prime}\left(\mathbb{X}_{s}^{\varepsilon}\right) \mathbb{V}_{s}^{\varepsilon} B_{s}^{\varepsilon} d s, \quad 0 \leq t \leq \tau^{\varepsilon}
$$

On the event $A_{1}^{\varepsilon}=\left\{\sup _{0 \leq t \leq M}\left|\sigma^{\prime}\left(\mathbb{X}_{t}^{\varepsilon}\right) \mathbb{V}_{t}^{\varepsilon} B_{t}^{\varepsilon}\right| \leq \frac{1}{2 \varepsilon}\right\}$, we have

$$
d \mathbb{X}_{t}^{\varepsilon} \geq\left(\frac{t}{2}+\varepsilon \sigma\left(\mathbb{X}_{t}^{\varepsilon}\right) B_{t}^{\varepsilon}\right) d t \geq\left(\frac{t}{2}-C \varepsilon \mathbb{X}_{t}^{\varepsilon}\left|B_{t}^{\varepsilon}\right|\right) d t, \quad 0 \leq t \leq \tau^{\varepsilon} \wedge M
$$

where $C$ here stands for the Lipschitz constant of $\sigma$. We deduce

$$
d \overline{\mathbb{X}}_{t}^{\varepsilon} \geq \frac{t}{2} \exp \left(C \varepsilon \int_{0}^{t}\left|B_{s}^{\varepsilon}\right| d s\right) d t, \quad \text { with } \quad \overline{\mathbb{X}}_{t}^{\varepsilon}=\mathbb{X}_{t}^{\varepsilon} \exp \left(C \varepsilon \int_{0}^{t}\left|B_{s}^{\varepsilon}\right| d s\right)
$$

for $0 \leq t \leq \tau^{\varepsilon} \wedge M$. Therefore, on $A_{1}^{\varepsilon}, \tau^{\varepsilon}$ must be greater than $\mathrm{M}$, so that the above expression holds up to time $\mathrm{M}$ (at least). We deduce $d \overline{\mathbb{X}}_{t}^{\varepsilon} \geq(t / 2) d t$, for $0 \leq t \leq M$, so that $\overline{\mathbb{X}}_{t}^{\varepsilon} \geq t^{2} / 4$, for $0 \leq t \leq M$.

Intersect now $A_{1}^{\varepsilon}$ with $A_{2}^{\varepsilon}=\left\{\sup _{0 \leq t \leq M}\left|B_{t}^{\varepsilon}\right| \leq \frac{1}{\varepsilon M}\right\}$. Then, on $A_{1}^{\varepsilon} \cap A_{2}^{\varepsilon}$,

$$
\mathbb{X}_{t}^{\varepsilon} \geq \frac{t^{2}}{4} \exp (-C), \quad 0 \leq t \leq M
$$

To complete the proof, it remains to notice (from a standard tightness argument) that $\mathbb{P}\left(A_{1}^{\varepsilon} \cap A_{2}^{\varepsilon}\right) \rightarrow 1$ as $\varepsilon \rightarrow 0$, uniformly in $\left(z^{1}, z^{2}\right) \in K$. (The proof when $\left(z^{1}, z^{2}\right)$ is in $\Gamma^{-}$is similar.)

We now come back to the case when the initial condition of the $4 \mathrm{D}$ system is $((1,-\sqrt{2}),(-1, \sqrt{2}))$. The second key lemma consists in expanding the difference process $\left(\mathbb{X}^{\varepsilon}, \mathbb{V}^{\varepsilon}\right)$ w.r.t. $\varepsilon$, up to $\tau^{\varepsilon}=\inf \left\{t \geq 0: \mathbb{X}_{t}^{\varepsilon} \leq 0\right\}$.

Lemma 8 There exist a family of $1 D$ Brownian motions $\left(\left(B_{t}^{\varepsilon}\right)_{t \geq 0}\right)_{\varepsilon>0}$ and a family of random continuous processes $\left(g^{\varepsilon}: \mathbb{R}_{+} \rightarrow \mathbb{R}\right)_{\varepsilon>0}$, such that

$$
\forall T>0, \quad \lim _{R \rightarrow+\infty} \sup _{0<\varepsilon \leq 1} \mathbb{P}\left\{\sup _{0 \leq t \leq T}\left|g_{t}^{\varepsilon}\right|>R\right\}=0,
$$

and the processes

$$
\begin{aligned}
& d \mathbb{V}_{t}^{(0)}=d t, \quad d \mathbb{X}_{t}^{(0)}=\mathbb{V}_{t}^{(0)} d t, \quad\left(\mathbb{X}_{0}^{(0)}, \mathbb{V}_{0}^{(0)}\right)=(1,-\sqrt{2}) \\
& d \mathbb{V}_{t}^{(1, \varepsilon)}=\sigma\left(\mathbb{X}_{t}^{(0)}\right) d B_{t}^{\varepsilon}, \quad d \mathbb{X}_{t}^{(1, \varepsilon)}=\mathbb{V}_{t}^{(1, \varepsilon)} d t, \quad\left(\mathbb{X}_{0}^{(1, \varepsilon)}, \mathbb{V}_{0}^{(1, \varepsilon)}\right)=(0,0),
\end{aligned}
$$

satisfy

$$
\left|\mathbb{X}_{t}^{\varepsilon}-\left(\mathbb{X}_{t}^{(0)}+\varepsilon \mathbb{X}_{t}^{(1, \varepsilon)}\right)\right|+\left|\mathbb{V}_{t}^{\varepsilon}-\left(\mathbb{V}_{t}^{(0)}+\varepsilon \mathbb{V}_{t}^{(1, \varepsilon)}\right)\right| \leq \varepsilon^{2}\left|g_{t}^{\varepsilon}\right|, \quad 0 \leq t \leq \tau^{\varepsilon}
$$


Proof. By Condition 4, we can write

$$
d \mathbb{V}_{t}^{\varepsilon}=d t+\varepsilon \sigma\left(\mathbb{X}_{t}^{\varepsilon}\right) d B_{t}^{\varepsilon}, \quad 0 \leq t \leq \tau^{\varepsilon},
$$

for some 1D Brownian motion $\left(B_{t}^{\varepsilon}\right)_{t \geq 0}$, so that

$$
d\left[\delta \mathbb{X}_{t}^{\varepsilon}\right]=\delta \mathbb{V}_{t}^{\varepsilon} d t, \quad d\left[\delta \mathbb{V}_{t}^{\varepsilon}\right]=\varepsilon\left[\sigma\left(\mathbb{X}_{t}^{\varepsilon}\right)-\sigma\left(\mathbb{X}_{t}^{(0)}\right)\right] d B_{t}^{\varepsilon}, \quad 0 \leq t \leq \tau^{\varepsilon},
$$

with $\delta \mathbb{X}_{t}^{\varepsilon}=\mathbb{X}_{t}^{\varepsilon}-\left(\mathbb{X}_{t}^{(0)}+\varepsilon \mathbb{X}_{t}^{(1, \varepsilon)}\right), \quad \delta \mathbb{V}_{t}^{\varepsilon}=\mathbb{V}_{t}^{\varepsilon}-\left(\mathbb{V}_{t}^{(0)}+\varepsilon \mathbb{V}_{t}^{(1, \varepsilon)}\right)$. We perform the same integration by parts as above, with

$$
\delta \overline{\mathbb{V}}_{t}^{\varepsilon}=\delta \mathbb{V}_{t}^{\varepsilon}-\varepsilon\left(\sigma\left(\mathbb{X}_{t}^{\varepsilon}\right)-\sigma\left(\mathbb{X}_{t}^{(0)}\right)\right) B_{t}^{\varepsilon} .
$$

Then, we can find a family of random continuous functions $\left(\left(v_{t}^{0, \varepsilon}\right)_{t \geq 0}\right)_{\varepsilon>0}$, satisfying (23), such that

$$
\begin{aligned}
d\left[\delta \overline{\mathbb{V}}_{t}^{\varepsilon}\right] & =-\varepsilon\left(\sigma^{\prime}\left(\mathbb{X}_{t}^{\varepsilon}\right) \mathbb{V}_{t}^{\varepsilon}-\sigma^{\prime}\left(\mathbb{X}_{t}^{(0)}\right) \mathbb{V}_{t}^{(0)}\right) B_{t}^{\varepsilon} d t \\
& =-\varepsilon\left(\sigma^{\prime}\left(\mathbb{X}_{t}^{\varepsilon}\right) \mathbb{V}_{t}^{\varepsilon}-\sigma^{\prime}\left(\mathbb{X}_{t}^{(0)}+\varepsilon \mathbb{X}_{t}^{(1, \varepsilon)}\right)\left(\mathbb{V}_{t}^{(0)}+\varepsilon \mathbb{V}_{t}^{(1, \varepsilon)}\right)\right) B_{t}^{\varepsilon} d t+\varepsilon^{2} v_{t}^{0, \varepsilon} d t \\
& =-\varepsilon \sigma^{\prime}\left(\mathbb{X}_{t}^{\varepsilon}\right) B_{t}^{\varepsilon} \delta \mathbb{V}_{t}^{\varepsilon} d t \\
& -\varepsilon\left(\sigma^{\prime}\left(\mathbb{X}_{t}^{\varepsilon}\right)-\sigma^{\prime}\left(\mathbb{X}_{t}^{(0)}+\varepsilon \mathbb{X}_{t}^{(1, \varepsilon)}\right)\right)\left(\mathbb{V}_{t}^{(0)}+\varepsilon \mathbb{V}_{t}^{(1, \varepsilon)}\right) B_{t}^{\varepsilon} d t+\varepsilon^{2} v_{t}^{0, \varepsilon} d t
\end{aligned}
$$

Since $\sigma^{\prime}$ is Lipschitz-continuous, we can find two families of random functions $\left(\left(v_{t}^{1, \varepsilon}\right)_{t \geq 0}\right)_{\varepsilon>0}$ and $\left(\left(v_{t}^{2, \varepsilon}\right)_{t \geq 0}\right)_{\varepsilon>0}$, satisfying (23), such that

$$
d\left[\delta \overline{\mathbb{V}}_{t}^{\varepsilon}\right]=\varepsilon v_{t}^{1, \varepsilon} \delta \mathbb{X}_{t}^{\varepsilon} d t+\varepsilon v_{t}^{2, \varepsilon} \delta \overline{\mathbb{V}}_{t}^{\varepsilon} d t+\varepsilon^{2} v_{t}^{0, \varepsilon} d t
$$

In a similar way, we can find two families of random functions $\left(\left(x_{t}^{0, \varepsilon}\right)_{t \geq 0}\right)_{\varepsilon>0}$ and $\left(\left(x_{t}^{1, \varepsilon}\right)_{t \geq 0}\right)_{\varepsilon>0}$, satisfying $(23)$, such that

$$
d\left[\delta \mathbb{X}_{t}^{\varepsilon}\right]=\varepsilon x_{t}^{1, \varepsilon} \delta \mathbb{X}_{t}^{\varepsilon} d t+\delta \overline{\mathbb{V}}_{t}^{\varepsilon} d t+\varepsilon^{2} x_{t}^{0, \varepsilon} d t
$$

Bounding the resolvent of the linear system (25-26) in terms of the bounds for $x^{1, \varepsilon}, v^{1, \varepsilon}$ and $v^{2, \varepsilon}$, the result easily follows.

\subsection{Proof of Proposition 6}

We emphasize that $\mathbb{V}_{t}^{(0)}=-\sqrt{2}+t$ and $\mathbb{X}_{t}^{(0)}=(1-t / \sqrt{2})^{2}$ and that

$$
\begin{aligned}
& \mathbb{V}_{t}^{(1, \varepsilon)}=\int_{0}^{t} \sigma\left(\mathbb{X}_{s}^{(0)}\right) d B_{s}^{\varepsilon}=\int_{0}^{t} \sigma\left[\left(1-\frac{s}{\sqrt{2}}\right)^{2}\right] d B_{s}^{\varepsilon} \\
& \mathbb{X}_{t}^{(1, \varepsilon)}=\int_{0}^{t} \int_{0}^{s} \sigma\left[\left(1-\frac{r}{\sqrt{2}}\right)^{2}\right] d B_{r}^{\varepsilon}=\int_{0}^{t}(t-r) \sigma\left[\left(1-\frac{r}{\sqrt{2}}\right)^{2}\right] d B_{r}^{\varepsilon}
\end{aligned}
$$

Choosing $t_{0}=\sqrt{2}$ and keeping in mind that $\sigma(1) \neq 0$, we deduce 
Lemma 9 The r.v.'s $\left(\mathbb{X}_{t_{0}}^{(1, \varepsilon)}\right)_{\varepsilon>0}$ have the same Gaussian law with zero mean and non-zero variance. In particular, $\mathbb{P}\left\{\mathbb{X}_{t_{0}}^{(1, \varepsilon)}>0\right\}=\mathbb{P}\left\{\mathbb{X}_{t_{0}}^{(1, \varepsilon)} \geq 0\right\}=1 / 2$.

We claim

Lemma 10 For any real $M>t_{0}=\sqrt{2}$ and any $\delta>0$,

$$
\begin{aligned}
\text { (i) } & \lim _{\varepsilon \rightarrow 0} \mathbb{P}\left(\left\{\tau^{\varepsilon} \leq M\right\} \cap\left\{\mathbb{X}_{t_{0}}^{(1, \varepsilon)}>\delta\right\}\right)=0, \\
\text { (ii) } & \lim _{\varepsilon \rightarrow 0} \mathbb{P}\left(\left\{\tau^{\varepsilon}>M\right\} \cap\left\{\mathbb{X}_{t_{0}}^{(1, \varepsilon)}<-\delta\right\}\right)=0, \\
\text { (iii) } & \lim _{\varepsilon \rightarrow 0} \mathbb{P}\left(\left\{\tau^{\varepsilon} \leq M\right\} \cap\left\{\tau^{\varepsilon} \notin\left(t_{0}-\delta, t_{0}+\delta\right)\right\}\right)=0 .
\end{aligned}
$$

Proof. Given $M>\sqrt{2}$, we set $E_{M}^{\varepsilon}=\left\{\tau^{\varepsilon} \leq M\right\}$. We know from Lemma 8 , that there exists a tight family of random variables $\left(\zeta_{M}^{\varepsilon}\right)_{0<\varepsilon \leq 1}$ such that

$$
\left|\mathbb{X}_{t}^{\varepsilon}-\left(\mathbb{X}_{t}^{(0)}+\varepsilon \mathbb{X}_{t}^{(1, \varepsilon)}\right)\right| \leq \varepsilon^{2} \zeta_{M}^{\varepsilon}, \quad 0 \leq t \leq \tau^{\varepsilon} \wedge M
$$

Therefore, on $E_{M}^{\varepsilon}$, we can choose $t=\tau^{\varepsilon}$ above. We deduce that

$$
\left|\left(1-\frac{\tau^{\varepsilon}}{\sqrt{2}}\right)^{2}+\varepsilon \mathbb{X}_{\tau^{\varepsilon}}^{(1, \varepsilon)}\right| \leq \varepsilon^{2} \zeta_{M}^{\varepsilon}
$$

Up to a modification of $\zeta_{M}^{\varepsilon}$, we deduce (which is $(i i i)$ )

$$
\left|\tau^{\varepsilon}-\sqrt{2}\right|^{2} \leq \varepsilon \zeta_{M}^{\varepsilon}
$$

We now prove $(i)$. From (29), we deduce that $\mathbb{X}_{\tau^{\varepsilon}}^{(1, \varepsilon)} \leq \varepsilon \zeta_{M}^{\varepsilon}$ on $E_{M}^{\varepsilon}$. Since $\mathbb{X}^{(1, \varepsilon)}$ is Lipschitz continuous on the interval $[0, M]$, we deduce from (30) that there exists a tight family of random variables $\left(C_{M}^{\varepsilon}\right)_{0<\varepsilon \leq 1}$ such that

$\mathbb{X}_{t_{0}}^{(1, \varepsilon)}=\mathbb{X}_{\tau^{\varepsilon}}^{(1, \varepsilon)}+\mathbb{X}_{t_{0}}^{(1, \varepsilon)}-\mathbb{X}_{\tau^{\varepsilon}}^{(1, \varepsilon)} \leq \varepsilon \zeta_{M}^{\varepsilon}+C_{M}^{\varepsilon}\left|\tau^{\varepsilon}-t_{0}\right| \leq \varepsilon \zeta_{M}^{\varepsilon}+C_{M}^{\varepsilon} \varepsilon^{1 / 2}\left(\zeta_{M}^{\varepsilon}\right)^{1 / 2}$

That is, for every $\delta>0, \lim _{\varepsilon \rightarrow 0} \mathbb{P}\left(E_{M}^{\varepsilon} \cap\left\{\mathbb{X}_{t_{0}}^{(1, \varepsilon)}>\delta\right\}\right)=0$.

We finally prove $(i i)$. From (28), we know that $\varepsilon \mathbb{X}_{t_{0}}^{(1, \varepsilon)} \geq \mathbb{X}_{t_{0}}^{\varepsilon}-\varepsilon^{2} \zeta_{t_{0}}^{\varepsilon}$. Therefore, on $\left(E_{M}^{\varepsilon}\right)^{\complement}, \varepsilon \mathbb{X}_{t_{0}}^{(1, \varepsilon)} \geq-\varepsilon^{2} \zeta_{t_{0}}^{\varepsilon}$. This proves that, for every $\delta>0$,

$$
\lim _{\varepsilon \rightarrow 0} \mathbb{P}\left(\left(E_{M}^{\varepsilon}\right)^{\complement} \cap\left\{\mathbb{X}_{t_{0}}^{(1, \varepsilon)}<-\delta\right\}\right)=0 .
$$




\section{Lemma 11 It holds}

$$
\begin{aligned}
& \text { (i) } \quad \forall M>\sqrt{2}, \quad \lim _{\varepsilon \rightarrow 0} \mathbb{P}\left\{\tau^{\varepsilon}>M\right\}=1 / 2, \\
& \text { (ii) } \quad \forall \delta>0, \quad \lim _{\varepsilon \rightarrow 0} \mathbb{P}\left\{\tau^{\varepsilon} \in\left(t_{0}-\delta, t_{0}+\delta\right)\right\}=1 / 2 .
\end{aligned}
$$

In particular, $\tau^{\varepsilon}$ converges in law towards $\frac{1}{2} \delta_{t_{0}}+\frac{1}{2} \delta_{+\infty}$.

Proof. From Lemmas 9 and 10, for any $M>\sqrt{2}$ and any $\delta>0$,

$$
\limsup _{\varepsilon \rightarrow 0} \mathbb{P}\left\{\tau^{\varepsilon}>M\right\} \leq \limsup _{\varepsilon \rightarrow 0} \mathbb{P}\left\{\mathbb{X}_{t_{0}}^{(1, \varepsilon)} \geq-\delta\right\}=\mathbb{P}\left\{\mathbb{X}_{t_{0}}^{(1,1)} \geq-\delta\right\} .
$$

Letting $\delta$ tend toward 0 , we obtain $\lim \sup _{\varepsilon \rightarrow 0} \mathbb{P}\left\{\tau^{\varepsilon}>M\right\} \leq 1 / 2$. Similarly,

$$
\limsup _{\varepsilon \rightarrow 0} \mathbb{P}\left\{\tau^{\varepsilon} \leq M\right\} \leq \mathbb{P}\left\{\mathbb{X}_{t_{0}}^{(1,1)} \leq 0\right\}=\frac{1}{2}
$$

We deduce $(i)$. Then, $($ ii $)$ follows from $($ iii $)$ in Lemma 10.

We finally claim

Lemma 12 Set $\sigma^{\varepsilon}=\inf \left\{t \geq 0: \mathbb{V}_{t}^{\varepsilon} \geq 0\right\}$. Then, for all $M>0$,

$$
\begin{aligned}
& \lim _{\varepsilon \rightarrow 0} \mathbb{P}\left\{\tau^{\varepsilon} \leq M, \sigma^{\varepsilon}<\tau^{\varepsilon}\right\}=0, \\
& \lim _{\varepsilon \rightarrow 0} \mathbb{P}\left\{\tau_{2}^{\varepsilon} \geq M\right\}=1 .
\end{aligned}
$$

Proof. By Lemma 11, we can assume $M>\sqrt{2}$. We then start with the proof of (31). By Markov property, we notice that

$$
\begin{aligned}
& \mathbb{P}\left\{\tau^{\varepsilon} \leq M, \sigma^{\varepsilon}<\tau^{\varepsilon}\right\} \\
& \leq \int_{\Gamma_{2}} \mathbf{1}_{\left\{\tilde{\pi}_{x}\left(z^{2}-z^{1}\right)>0, \tilde{\pi}_{v}\left(z^{2}-z^{1}\right)=0\right\}} \mathbb{P}\left\{\tau^{\varepsilon} \leq M \mid\left(Z_{0}^{1, \varepsilon}, Z_{0}^{2, \varepsilon}\right)=\left(z^{1}, z^{2}\right)\right\} d \eta^{\varepsilon}\left(z^{1}, z^{2}\right),
\end{aligned}
$$

where $\eta^{\varepsilon}$ is the conditional law of $\left(Z_{\rho^{\varepsilon}}^{1, \varepsilon}, Z_{\rho^{\varepsilon}}^{2, \varepsilon}\right)$ given $\rho^{\varepsilon} \leq M$, with $\rho^{\varepsilon}=$ $\inf \left(\sigma^{\varepsilon}, \tau^{\varepsilon}\right)$, under the initial condition $((1,-\sqrt{2}),(-1, \sqrt{2}))$. Using $(i)$ in Lemma 11, it is plain to see that the distributions $\left(\eta^{\varepsilon}\right)_{0<\varepsilon \leq 1}$ are tight. By Lemma 7, this shows (31). Similarly, we have

$$
\begin{aligned}
& \mathbb{P}\left\{\tau_{2}^{\varepsilon} \leq M, \tau^{\varepsilon}<\sigma^{\varepsilon}\right\} \\
& \leq \int_{\Gamma_{2}} \mathbf{1}_{\left\{\tilde{\pi}_{x}\left(z^{2}-z^{1}\right)=0, \tilde{\pi}_{v}\left(z^{2}-z^{1}\right)<0\right\}} \mathbb{P}\left\{\tau^{\varepsilon} \leq M \mid\left(Z_{0}^{1, \varepsilon}, Z_{0}^{2, \varepsilon}\right)=\left(z^{1}, z^{2}\right)\right\} d \eta^{\varepsilon}\left(z^{1}, z^{2}\right),
\end{aligned}
$$

which tends to 0 by the same argument as above. Since $\lim _{\varepsilon \rightarrow 0} \mathbb{P}\left\{\tau_{2}^{\varepsilon} \leq\right.$ $\left.M, \sigma^{\varepsilon}<\tau^{\varepsilon}\right\} \leq \lim _{\varepsilon \rightarrow 0} \mathbb{P}\left\{\tau^{\varepsilon} \leq M, \sigma^{\varepsilon}<\tau^{\varepsilon}\right\}=0$, we deduce (32). (Keep in mind that $\mathbb{P}\left\{\tau^{\varepsilon}=\sigma^{\varepsilon}\right\}=0$.) 


\section{Structure of the noise}

In this section, we investigate the meaning of Assumption (A.4). First, we translate it into an ellipticity property of the noise. Second, we discuss some general examples inspired from turbulence theory. Finally, we prove that ellipticity of the noise lifts up to hypoellipticity of any mollified version of Eq. (13). In the whole section, we shall make use of the notations in (14).

\subsection{Ellipticity of the noise}

When $F=0$ and $x \in \Gamma_{x, N}^{\complement}$, Span $\left\{A_{k}(x)\right\}_{k \in \mathbb{N} \backslash\{0\}} \subsetneq \mathbb{R}^{N d}$ so that the velocity component in (13) moves along a restricted number of directions only. On the contrary, when $x \in \Gamma_{x, N}$, the noise generated at $x$ is non-degenerate because of the strict positivity of $Q(\tilde{x}, \tilde{y})$ on $\Gamma_{x, N}$ in (A.4):

Lemma $13 Q$ satisfies (A.4) if and only if

$$
\operatorname{Span}\left\{A_{k}(x)\right\}_{k \in \mathbb{N} \backslash\{0\}}=\mathbb{R}^{N d}, \quad \forall x=\left(x^{1}, \ldots, x^{N}\right) \in \Gamma_{x, N} .
$$

Proof. For any $v=\left(v^{1}, \ldots, v^{N}\right) \in \mathbb{R}^{N d} \backslash\{0\}$, we have $\sum_{k=1}^{\infty}\left\langle A_{k}(x), v\right\rangle_{\mathbb{R}^{N d}}^{2}=$ $\sum_{k=1}^{\infty}\left(\sum_{i=1}^{N}\left\langle\sigma_{k}\left(x^{i}\right), v^{i}\right\rangle_{\mathbb{R}^{d}}\right)^{2}=\sum_{i, j=1}^{N}\left\langle Q\left(x^{j}, x^{i}\right) v^{i}, v^{j}\right\rangle_{\mathbb{R}^{d}}>0$

Below, we exhibit interesting examples of strictly positive covariance functions $Q(\tilde{x}, \tilde{y})$ that are space-homogeneous. Precisely, we shall assume that there is a symmetric $d \times d$ matrix-valued function $Q(\tilde{x})$, such that $Q(\tilde{x}, \tilde{y})=Q(\tilde{x}-\tilde{y})=Q(\tilde{y}-\tilde{x})$, with the following spectral representation:

$$
Q(\tilde{x})=\int_{\mathbb{R}^{d}} e^{i k \cdot \tilde{x}} \mathcal{Q}(k) d k, \quad \tilde{x} \in \mathbb{R}^{d},
$$

where the spectral density $\mathcal{Q}$ takes values in the space of non-negative real symmetric $d \times d$ matrices, with coordinates in $L^{1}\left(\mathbb{R}^{d}\right)$, and satisfies $\mathcal{Q}(-k)=$ $\mathcal{Q}(k), k \in \mathbb{R}^{d}$. (Above, $k \cdot \tilde{x}$ is a shorten notation for $\langle k, \tilde{x}\rangle_{\mathbb{R}^{d}}$.) In this framework, we have the general criterion:

Lemma 14 Assume that $\mathcal{Q}$ has the following property: for any $\mathbb{R}^{d}$-valued trigonometric polynomial $v(k)$ of the form $v(k)=\sum_{j=1}^{N} v^{j} e^{i k \cdot x^{j}}$, for some $\left(x^{1}, \ldots, x^{N}\right),\left(v^{1}, \ldots, v^{N}\right) \in \mathbb{R}^{N d}$ and for $i^{2}=-1$, the a.e. equality

$$
\langle\mathcal{Q}(k) v(k), v(k)\rangle_{\mathbb{C}^{d}}=0 \quad \text { for a.e. } k \in \mathbb{R}^{d}
$$


implies $v(k)=0$ for any $k \in \mathbb{R}^{d}$, where $\langle\cdot, \cdot\rangle_{\mathbb{C}^{d}}$ denotes the Hermitian product in $\mathbb{C}^{d}$. Then $Q(\tilde{x}, \tilde{y})$ is strictly positive on $\Gamma_{x, N}$. (Keep in mind that, for any $u, u^{\prime} \in \mathbb{C}^{d},\left\langle u, u^{\prime}\right\rangle_{\mathbb{C}^{d}}=\sum_{j=1}^{d} \bar{u}^{j}\left(u^{\prime}\right)^{j}, \bar{u}$ denoting the conjugate of $u$. We will also write $\left\langle u, u^{\prime}\right\rangle_{\mathbb{C}^{d}}=\left\langle\bar{u}, u^{\prime}\right\rangle_{\mathbb{R}^{d}}$ with an abuse of notation.)

Proof. The proof follows from the identity:

$$
\sum_{j, \ell=1}^{N}\left\langle Q\left(x^{j}, x^{\ell}\right) v^{\ell}, v^{j}\right\rangle_{\mathbb{R}^{d}}=\int_{\mathbb{R}^{d}}\langle\mathcal{Q}(k) v(k), v(k)\rangle_{\mathbb{C}^{d}} d k
$$

where $v(k)=\sum_{j=1}^{N} v^{j} e^{i k \cdot x^{j}}$. Indeed, $v(k)=0$ for any $k \in \mathbb{R}^{d}$ implies $\left(v^{1}, \ldots, v^{N}\right)=0$ since $v(k)$ is a (vector valued) trigonometric polynomial driven by pairwise different vectors $x^{1}, \ldots, x^{N}$. (See Remark 15 below.)

Remark 15 Let $f: \mathbb{R}^{d} \rightarrow \mathbb{C}$ be of the form

$$
f(k)=\sum_{j=1}^{N}\left(a^{j}+\left\langle k, v^{j}\right\rangle_{\mathbb{C}^{d}}\right) e^{i x^{j} \cdot k}, \quad k \in \mathbb{R}^{d},
$$

where $a^{j} \in \mathbb{C}, v^{j} \in \mathbb{C}^{d}$ and $\left(x^{1}, \ldots, x^{N}\right) \in \Gamma_{x, N}$. If there is a Borel set $A \subset \mathbb{R}^{d}$ of positive Lebesgue measure such that $f=0$ on $A$, then $a_{j}=0$ and $v_{j}=0$ for any $j=1, \ldots, N$. Indeed, by a standard extension of the principle of analytic continuation, $f(k)=0$ for any $k \in \mathbb{R}^{d}$. Given a smooth function $\varphi: \mathbb{R}^{d} \rightarrow \mathbb{C}$ with compact support, we denote by $\hat{\varphi}$ its Fourier transform. We have $\int_{\mathbb{R}^{d}} f(k) \hat{\varphi}(k) d k=0$ and thus $\sum_{j=1}^{N}\left[a^{j} \varphi\left(x^{j}\right)-i\left\langle v^{j}, \nabla \varphi\left(x^{j}\right)\right\rangle_{\mathbb{R}^{d}}\right]=0$. Since the points $x^{i}$ are all different, we may construct a function $\varphi$ such that $\varphi\left(x^{j}\right)=\bar{a}^{j}$ and $\nabla \varphi\left(x^{j}\right)=i \bar{v}^{j}$.

By Lemma 14 and Remark 15, we get as a first example:

Proposition 16 If $\mathcal{Q}(k)$ is strictly positive definite on a Borel subset of $\mathbb{R}^{d}$ of positive Lebesgue measure, then $Q(\tilde{x}, \tilde{y})$ is strictly positive on $\Gamma_{x, N}$.

\subsection{Isotropic random fields}

Proposition 16 does not cover important examples, as the following one, which appears in the literature about diffusion of passive scalars: 
Example 17 We say that $Q$ is isotropic if $Q(U \tilde{x})=U Q(\tilde{x}) U^{\top}$, for any $\tilde{x} \in \mathbb{R}^{d}$ and $U \in \mathcal{O}\left(\mathbb{R}^{d}\right)$, where $\mathcal{O}\left(\mathbb{R}^{d}\right)$ is the set of orthogonal matrices of dimension $d\left(U^{\top}\right.$ denotes the transpose of $\left.U\right)$. This is the case when $\mathcal{Q}(k)$ in (34) has the form

$$
\mathcal{Q}(k)=\pi_{k} f(|k|), \quad \text { that is } \quad Q(x)=\int_{\mathbb{R}^{d}} e^{i k \cdot x} \pi_{k} f(|k|) d k,
$$

where $\pi_{k}=1$, if $d=1$, and $\pi_{k}=(1-\mathrm{p}) I_{d}+|k|^{-2}(\mathrm{p} d-1) k \otimes k$ for some $\mathrm{p} \in[0,1]$, if $d \geq 2$, and $f:[0,+\infty) \rightarrow \mathbb{R}$ is in $L^{1}([0,+\infty))$ and satisfies

$$
f(r) \geq 0 \quad \text { for a.e. } r>0 \text {. }
$$

The matrix $\mathcal{Q}(k)$ is symmetric, it satisfies $\mathcal{Q}(-k)=\mathcal{Q}(k)$, and it is almosteverywhere non-negative because (we restrict the proof to $d \geq 2$ )

$$
\begin{aligned}
|k|^{2}\left\langle\pi_{k} w, w\right\rangle_{\mathbb{C}^{d}} & =(1-\mathrm{p})|k|^{2}|w|^{2}+(\mathrm{p} d-1)\left|\langle k, w\rangle_{\mathbb{C}^{d}}\right|^{2} \\
& \geq(1-\mathrm{p})\left|\langle k, w\rangle_{\mathbb{C}^{d}}\right|^{2}+(\mathrm{p} d-1)\left|\langle k, w\rangle_{\mathbb{C}^{d}}\right|^{2} \\
& =\mathrm{p}(d-1)\left|\langle k, w\rangle_{\mathbb{C}^{d}}\right|^{2} \geq 0,
\end{aligned}
$$

(Inequality is here given in $\mathbb{C}^{d}$ but only the $\mathbb{R}^{d}$ part is useful to prove nonnegativity of $\mathcal{Q}(k)$. The full inequality in $\mathbb{C}^{d}$ will be used next.)

We refer to [22], [29] and [33] for references where this form (for particular choices of $f$ ) is used or investigated. This class of covariances is related to the Batchelor regime of the Kraichnan model, where $f(r)=\left(r_{0}^{2}+r^{2}\right)^{-(d+\varpi) / 2}$ with $\varpi=2$, see [11]. In the limit $r_{0} \rightarrow 0$, the covariance of the increments of the noise is scale invariant with scaling exponent equal to 2. The "turbulent regime" of the Kraichnan model $(0 \leq \varpi<2)$ is in contrast not included in our main final result because of the regularity properties we require on $Q$.

Proposition 18 If there exists a Borel set $A \subset[0, \infty)$ such that $\operatorname{Leb}_{1}(A)>0$ and $f(r)>0$ for $r \in A$, then $Q(\tilde{x}, \tilde{y})$ is strictly positive on $\Gamma_{x, N}$.

Proof. Step 1. From Lemma 14 it is sufficient to prove that the condition

$$
f(|k|)\left\langle\pi_{k} v(k), v(k)\right\rangle_{\mathbb{C}^{d}}=0 \quad \text { for a.e. } k \in \mathbb{R}^{d}
$$

implies $v(k)=0$ for any $k \in \mathbb{R}^{d}$, when $v(k)$ has the form $v(k)=\sum_{j=1}^{N} v^{j} e^{i k \cdot x^{j}}$ for some $\left(v^{1}, \ldots, v^{N}\right) \in \mathbb{R}^{N d}$ and $\left(x^{1}, \ldots, x^{N}\right) \in \Gamma_{x, N}$. Since $f \neq 0$ on $A$, it 
holds $\left\langle\pi_{k} v(k), v(k)\right\rangle_{\mathbb{C}^{d}}=0$ for $k$ in a Borel subset $A^{*} \subset \mathbb{R}^{d}$ of positive measure. We now prove that this implies $v(k) \equiv 0$.

We focus on the condition $\left\langle\pi_{k} w, w\right\rangle_{\mathbb{C}^{d}}=0$ for some $w \in \mathbb{C}^{d}$. When $d=1$, it implies $w=0$. For $\mathrm{p} \in(0,1], d>1$, inequality (37) implies $\mathrm{p}(d-1)\langle k, w\rangle_{\mathbb{C}^{d}}^{2}=0$, and thus $\langle k, w\rangle_{\mathbb{C}^{d}}^{2}=0$. Finally, in the case $\mathrm{p}=0$, $d>1$, for all $w \in \mathbb{C}^{d}$ we have

$$
|k|^{2}\left\langle\pi_{k} w, w\right\rangle_{\mathbb{C}^{d}}=\left(|k||w|-\langle k, w\rangle_{\mathbb{C}^{d}}\right)^{2}
$$

and thus $\left\langle\pi_{k} w, w\right\rangle_{\mathbb{C}^{d}}=0$ implies that $w=\lambda k$, for some $\lambda \in \mathbb{R}$, if $k \neq 0$.

Going back to the main line of the proof, we have $\left\langle\pi_{k} v(k), v(k)\right\rangle_{\mathbb{C}^{d}}=0$ for all $k \in A^{*}$. Depending on the values of $\mathrm{p}$ and $d$, this implies at least one of the three following conditions: $v(k)=0$ for all $k \in A^{*}$, or $\langle k, v(k)\rangle_{\mathbb{C}^{d}}=0$ for all $k \in A^{*}$, or $v(k) \| k$ for all $k \in A^{*}$ (except maybe at $k=0$ ). By Remark 15, $v j=0$, for $j=1, \ldots, N$, in the two first cases. In the third case, we notice that $v(k) \| k$ may be written as $\sum_{j=1}^{N}\left\langle v_{\ell}^{j} e_{\ell^{\prime}}-v_{\ell^{\prime}}^{j} e_{\ell}, k\right\rangle_{\mathbb{C}^{d}} e^{i x^{j} \cdot k}=0$, for $1 \leq \ell, \ell^{\prime} \leq d$, where $\left(e_{\ell}\right)_{1 \leq \ell \leq d}$ is the canonical basis of $\mathbb{C}^{d}$. By Remark 15 again, this also implies $v^{j}=0$, for $j=1, \ldots, N$. (See also [17, Theorem 4.7] and [10].)

We are now able to give examples for which Theorem 2 applies:

Proposition 19 In the case when $d=1$, consider $Q$ as in Example 17, with $f \in L^{1}\left(\mathbb{R}_{+}, \mathbb{R}_{+}\right)$satisfying the assumption of Proposition 18 together with $\int_{0}^{+\infty} k^{4} f(k) d k<+\infty$, then Conditions 3 and 4 in Section 2 are satisfied with $\sigma(x)=\operatorname{sign}(x) \sqrt{(Q(0)-Q(x)) / 2}$, for $x \in \mathbb{R}$.

Proof. Go back to the framework of Section 2 and recall (16) and (17). Existence and uniqueness in Condition 3 follow from Theorem 22 below. In order to prove (18), we consider an arbitrary random initial condition $\left(Z_{0}^{1, \varepsilon}, Z_{0}^{2, \varepsilon}\right) \in \Gamma_{2}$, independent of the noise $\left(\left(W_{t}^{k}\right)_{t \geq 0}\right)_{k \geq 1}$. For any $t \geq 0$,

$$
\begin{aligned}
d \mathbb{X}_{t}^{\varepsilon} & =\operatorname{sign}\left(\mathbb{X}_{t}^{\varepsilon}\right) d t+\varepsilon \sum_{k \geq 1} \frac{\sigma_{k}\left(X_{t}^{2, \varepsilon}\right)-\sigma_{k}\left(X_{t}^{1, \varepsilon}\right)}{2} d W_{t}^{k} \\
& =\operatorname{sign}\left(\mathbb{X}_{t}^{\varepsilon}\right)\left(d t+\varepsilon \sqrt{\rho_{t}^{\varepsilon}} d B_{t}^{\varepsilon}\right),
\end{aligned}
$$

with $\rho_{t}^{\varepsilon}=Q(0)-Q\left(\mathbb{X}_{t}^{\varepsilon}\right) \geq 0$ and

$$
\begin{aligned}
d B_{t}^{\varepsilon}= & \left(\mathbf{1}_{\left\{\mathbb{X}_{t}^{\varepsilon} \geq 0\right\}}-\mathbf{1}_{\left\{\mathbb{X}_{t}^{\varepsilon}<0\right\}}\right) \\
& \times\left(\sum_{k \geq 1} \mathbf{1}_{\left\{\rho_{t}^{\varepsilon}>0\right\}} \frac{\sigma_{k}\left(X_{t}^{2, \varepsilon}\right)-\sigma_{k}\left(X_{t}^{1, \varepsilon}\right)}{\sqrt{2 \rho_{t}^{\varepsilon}}} d W_{t}^{k}+\mathbf{1}_{\left\{\rho_{t}^{\varepsilon}=0\right\}} d W_{t}^{1}\right) .
\end{aligned}
$$


It is well-checked that $d\left\langle B^{\varepsilon}\right\rangle_{t}=d t$. By Lévy's Theorem, $\left(B_{t}^{\varepsilon}\right)_{t \geq 0}$ is a Brownian motion w.r.t. the augmented filtration generated by the initial condition $\left(Z_{0}^{1, \varepsilon}, Z_{0}^{2, \varepsilon}\right)$ and by the noise $\left(\left(W_{t}^{k}\right)_{t \geq 0}\right)_{k \geq 1}$.

We now investigate the properties of $\sigma$. Clearly, $\sigma(0)=0$. Below, we prove that $\sigma$ is $\mathcal{C}^{2}$ with bounded derivatives and that $\sigma(1)>0$. We have

$$
Q(0)-Q(x)=x^{2} \int_{\mathbb{R}} \frac{1-\cos (k x)}{x^{2}} f(|k|) d k=x^{2} \int_{\mathbb{R}} k^{2} \varphi(k x) f(|k|) d k,
$$

with $\varphi(u)=u^{-2}(1-\cos (u))$. Clearly, $\varphi$ is infinitely differentiable with bounded derivatives. Therefore, the function $\Phi: \mathbb{R} \ni x \mapsto \int_{\mathbb{R}} k^{2} \varphi(k x) f(|k|) d k$ is twice continuously differentiable with bounded derivatives. At $x=0$, $\Phi(0)>0$, so that $\sqrt{\Phi}$ is twice continuously differentiable in the neighborhood of 0 . Then, the function $\sigma$, which reads $\sigma(x)=x \sqrt{\Phi(x)}$, for $x \in \mathbb{R}$, is twice continuously differentiable in the neighborhood of 0 . Away from 0 , the function $\mathbb{R} \ni x \mapsto Q(0)-Q(x)$ has positive values so that its square root is also twice continuously differentiable and $\sigma$ is twice continuously differentiable as well. The derivatives of order one and two of $\sigma$ are bounded since the derivatives of order one and two of $Q$ are bounded and $Q(0)-Q(x) \rightarrow Q(0)>0$ as $|x| \rightarrow+\infty$. Moreover, $\sigma(1)$ is clearly positive.

\subsection{Hypoellipticity of the $N$-point motion}

Ellipticity of the noise turns into hypoellipticity of the system, in the following sense (the proof is standard and is thus left to the reader):

Proposition 20 Assume that, $F$ and $\sigma_{k}$, for any $k \in \mathbb{N} \backslash\{0\}$, are of class $\mathcal{C}^{1}$ on $\mathbb{R}^{d}$ and that, for every $x=\left(x^{1}, \ldots, x^{N}\right) \in \Gamma_{x, N}$, Span $\left\{A_{k}(x)\right\}_{k \in \mathbb{N} \backslash\{0\}}=$ $\mathbb{R}^{N d}$. Then, for every $z \in \mathbb{R}^{2 N d}$ of the form $z=(x, v)$ with $x=\left(x^{1}, \ldots, x^{N}\right) \in$ $\Gamma_{x, N}, v \in \mathbb{R}^{N d}$, we have $\operatorname{Span}\left\{\mathbb{A}_{k}(z),\left[\mathbb{A}_{k}, \mathbb{F}\right](z)\right\}_{k \in \mathbb{N} \backslash\{0\}}=\mathbb{R}^{2 N d}$. (Here, $[\cdot, \cdot]$ stands for the Lie bracket of vector fields.)

Here is the precise formulation of hypoellipticity in our framework:

Proposition 21 In addition to (A.1-4), assume that $F$ is Lipschitz continuous on the whole $\mathbb{R}^{d}$. Then, for every initial condition $Z^{0}=z \in \mathbb{R}^{2 N d}$, Eq. (13) admits a unique strong solution. Moreover, the mappings $\varphi_{t}: \mathbb{R}^{2 N d} \ni$ $z \mapsto Z_{t}$ subject to $Z_{0}=z, t \geq 0$, form a stochastic flow of homeomorphisms on $\mathbb{R}^{2 N d}$. Finally, for any $t>0$, the marginal law of the $2 \mathrm{Nd}$-dimensional vector $Z_{t}$ is absolutely continuous with respect to the Lebesgue measure when $z=(x, v)$ satisfies $x \in \Gamma_{x, N}$. 
Proof. Unique strong solvability and homeomorphism property of the flow may be found in [30] and [20, Chapter 4, Section 5]. When the coefficients $\left(\sigma_{k}\right)_{k \in \mathbb{N} \backslash\{0\}}$ are smooth, with derivatives of any order in $\ell^{2}(\mathbb{N} \backslash\{0\})$, and $\mathbb{F}$ is smooth as well, absolute continuity then follows from Proposition 20 and a suitable version of Hörmander's Theorem for systems driven by an infinite-dimensional noise. See for example [30, Theorem 4.3].

Here the coefficients are not smooth. Anyhow, absolute continuity follows from the Bouleau and Hirsch criterion directly. By Proposition 2.2 in [30], $\left(Z_{t}\right)_{t \geq 0}$ is differentiable in the sense of Malliavin with $\sum_{k=1}^{+\infty} \mathbb{E} \int_{0}^{t}\left|D_{s}^{k} Z_{t}\right|^{2} d s<$ $+\infty$, for any $t \geq 0$. We also know that

$$
D_{r}^{k} Z_{t}=Y_{t}\left(Y_{r}\right)^{-1} \mathbb{A}_{k}\left(Z_{r}\right), \quad 0 \leq r \leq t
$$

the equality holding true in $\mathbb{R}^{2 N d}$, where $\left(Y_{t}\right)_{t \geq 0}$ is an $\mathbb{R}^{2 N d \times 2 N d}$-valued process, solution a linear SDE of the form

$$
Y_{t}=I_{2 N d}+\sum_{k=1}^{+\infty} \int_{0}^{t} \alpha_{k}(s) Y_{s} d W_{s}^{k}+\int_{0}^{t} \alpha_{0}(s) Y_{s} d s, \quad t \geq 0
$$

the processes $\left(\alpha_{k}(s)\right)_{s \geq 0}, k \in \mathbb{N}$, being bounded and progressively-measurable and the infinite-dimensional process $\left(\left(\left|\alpha_{k}(s)\right|\right)_{s \geq 0}\right)_{k \in \mathbb{N} \backslash\{0\}}$ being bounded in $\ell^{2}(\mathbb{N} \backslash\{0\})$. When the coefficients $\mathbb{F}$ and $\mathbb{A}_{k}, k \in \mathbb{N} \backslash\{0\}$, in the compact formulation (14) are smooth, it holds $\alpha_{0}(s)=\nabla \mathbb{F}\left(Z_{s}\right)$ and $\alpha_{k}(s)=\nabla \mathbb{A}_{k}\left(Z_{s}\right)$, $k \in \mathbb{N} \backslash\{0\}$. We then use the following notation: given a square matrix $M$ of size $2 N d \times 2 N d$, we denote by $[M]_{x, x},[M]_{x, v},[M]_{v, x}$ and $[M]_{v, v}$ the blocks of size $N d \times N d$ corresponding to the decomposition of a vector $z \in \mathbb{R}^{2 N d}$ into coordinates $x=\Pi_{x}(z)$ and $v=\Pi_{v}(z)$ in $\mathbb{R}^{N d}$. With this notation, $\left[\alpha_{k}(s)\right]_{x, v}$ and $\left[\alpha_{k}(s)\right]_{v, v}$ are zero since $\mathbb{A}_{k}$ is independent of $v$. Similarly, $\left[\alpha_{k}(s)\right]_{x, x}$ is zero since $\Pi_{x}\left(\mathbb{A}_{k}\right) \equiv 0$ and $\left[\alpha_{0}(s)\right]_{x, x}=\left[\alpha_{0}(s)\right]_{v, v}=0$ since $\Pi_{x}(\mathbb{F}) \equiv v$ and $\Pi_{v}(\mathbb{F})$ is independent of $x$. Moreover, $\left[\alpha_{0}(s)\right]_{x, v}=I_{N d}$. These relationships keep true in the Lipschitz setting by a mollification argument. Finally, as in the finite-dimensional framework, we can check that, a.s., for any $t>0, Y_{t}$ is invertible, the inverse being of finite polynomial moments of any order.

For $r$ small, $Y_{r}=I_{2 N d}+o_{r}(1), o_{r}(1)$ standing for the Landau notation and almost-surely converging to 0 with $r$. Therefore, by the equalities $\left[\alpha_{0}(s)\right]_{x, x}=$ $\left[\alpha_{k}(s)\right]_{x, x}=\left[\alpha_{k}(s)\right]_{x, v}=\left[\alpha_{0}(s)\right]_{v, v}=\left[\alpha_{k}(s)\right]_{v, v}=0$ and $\left[\alpha_{0}(s)\right]_{x, v}=I_{N d}$, we deduce that $\left[Y_{r}\right]_{x, v}=I_{N d}+r o_{r}(1)$ and $\left[Y_{r}\right]_{v, v}=I_{N d}+o_{r}(1)$ and that $\left[Y_{r}\right]_{x, v}$ may expanded as

$$
\left[Y_{r}\right]_{x, v}=r I_{N d}+r o_{r}(1)
$$


Setting $\mathcal{Z}_{r}=\left(Y_{r}\right)^{-1} \mathbb{A}_{k}\left(Z_{r}\right) \in \mathbb{R}^{2 N d}$ and writing $\mathcal{Z}_{r}$ under the form $\mathcal{Z}_{r}=$ $\left(\left(\mathcal{X}_{r}^{i}, \mathcal{V}_{r}^{i}\right)\right)_{1 \leq i \leq N}$, we have $Y_{r} \mathcal{Z}_{r}=\mathbb{A}_{k}\left(Z_{r}\right)$, so that

$$
\left[Y_{r}\right]_{x, x} \mathcal{X}_{r}+\left[Y_{r}\right]_{x, v} \mathcal{V}_{r}=0, \quad\left[Y_{r}\right]_{v, x} \mathcal{X}_{r}+\left[Y_{r}\right]_{v, v} \mathcal{V}_{r}=A_{k}\left(X_{r}\right)
$$

that is $\mathcal{X}_{r}+r \mathcal{V}_{r}=r o_{r}(1)$, and $o_{r}(1) \mathcal{X}_{r}+\mathcal{V}_{r}=A_{k}\left(X_{r}\right)+o_{r}(1)$. We deduce $\mathcal{V}_{r}=A_{k}\left(X_{r}\right)+o_{r}(1)$ and $\mathcal{X}_{r}=r A_{k}\left(X_{r}\right)+r o_{r}(1)$, so that, by (38),

$$
\left(Y_{t}\right)^{-1} D_{r}^{k} Z_{t}=\left(r A_{k}(x)+r o_{r}(1), A_{k}(x)+o_{r}(1)\right)
$$

(The above equality holds almost-surely, $o_{r}(1)$ being random itself.) For a given $\omega \in \Omega$ for which (40) holds true, consider $\zeta=\left(\left(\chi^{i}, \nu^{i}\right)\right)_{1 \leq i \leq N} \in \mathbb{R}^{2 N d}$ such that $\left\langle D_{r}^{k} Z_{t}, \zeta\right\rangle_{\mathbb{R}^{d}}=0$ for any $0 \leq r \leq t$ and $k \in \mathbb{N} \backslash\{0\}$. Changing $\zeta$ into $\left(\left(Y_{t}\right)^{-1}\right)^{\top} \zeta$, we deduce from (40) that

$$
r \sum_{i=1}^{N}\left\langle\sigma_{k}\left(x^{i}\right), \chi^{i}\right\rangle_{\mathbb{R}^{d}}+\sum_{i=1}^{N}\left\langle\sigma_{k}\left(x^{i}\right), \nu^{i}\right\rangle_{\mathbb{R}^{d}}=r o_{r}(1)|\chi|+o_{r}(1)|\nu|,
$$

Letting $r \rightarrow 0$, we get $\nu \perp A_{k}(x)$ for any $k \in \mathbb{N} \backslash\{0\}$. By (A.4), $\nu=0$. Dividing the above equality by $r$ and letting $r \rightarrow 0, \chi=0$. We complete the proof by Bouleau and Hirsch criterion, see [31, Theorem 2.1.2].

\section{No coalescence of the stochastic dynamics}

We now prove the main result of the paper:

Theorem 22 Under (A.1-4), for any $z \in \Gamma_{N}$, there exists a unique solution $\left(Z_{t}(z)\right)_{t \geq 0}$ to (13) with $z$ as initial condition. It satisfies $\mathbb{P}\left\{\forall t \geq 0, Z_{t}(z) \in\right.$ $\left.\Gamma_{N}\right\}=1$ and $\mathbb{P}\left\{\operatorname{Leb}_{1}\left\{t \geq 0: \Pi_{x}\left(Z_{t}(z)\right) \in \Gamma_{x, N}^{\complement}\right\}=0\right\}=1$.

The proof is split into three parts: we first establish a priori estimates for a regularized version of (13); using a compactness argument, we deduce that strong unique solvability holds for Lebesgue almost-every starting point; taking advantage of the absolute continuity of the marginal laws of the regularized system, we establish strong unique solvability for any $z \in \Gamma_{N}$. 


\subsection{Smoothed system of equations}

For every $\varepsilon>0$, let $F_{\varepsilon}: \mathbb{R}^{d} \rightarrow \mathbb{R}^{d}$ be equal to $F$ outside $B_{d}(0, \varepsilon)$, but be smooth inside $B_{d}(0, \varepsilon)$, with $\sup _{x \in \mathbb{R}^{d}}\left|F_{\varepsilon}(x)\right| \leq \sup _{x \in \mathbb{R}^{d}}|F(x)|+1$. Given such an $F_{\varepsilon}$, we consider Eq. (13), but with $F_{\varepsilon}$ instead of $F$ therein (or, equivalently, the compact writing (14) when driven by $\mathbb{F}_{\varepsilon}$, with an appropriate definition of $\mathbb{F}_{\varepsilon}$ in (15)). By Proposition 21, the smoothed system is uniquely solvable for every initial condition in $\mathbb{R}^{2 N d}$, the solution being generically denoted by $\left(Z_{t}^{\varepsilon}=\left(X_{t}^{\varepsilon}, V_{t}^{\varepsilon}\right)\right)_{t \geq 0}$, with $X_{t}^{\varepsilon}=\left(X_{t}^{i, \varepsilon}\right)_{1 \leq i \leq N}$ and $V_{t}^{\varepsilon}=\left(V_{t}^{i, \varepsilon}\right)_{1 \leq i \leq N}$, and the associated flow by $\varphi_{t}^{\varepsilon}: \mathbb{R}^{2 N d} \ni z \mapsto Z_{t}^{\varepsilon}$ with $Z_{0}^{\varepsilon}=z, t \geq 0$.

By the a.e. equality $\operatorname{div}_{(x, v)} \mathbb{F}_{\varepsilon}=0$ and $\operatorname{div}_{(x, v)} \mathbb{A}_{k}=0$ for all $k \in \mathbb{N}$ (the divergence being here computed in the phase space), we get directly:

Lemma 23 For any $t \geq 0, \varphi_{t}^{\varepsilon}(\cdot)$ preserves the Lebesgue measure, that is, for all measurable and non negative $g, \mathbb{E} \int_{\mathbb{R}^{2 N d}} g\left(\varphi_{t}^{\varepsilon}(z)\right) d z=\int_{\mathbb{R}^{2 N d}} g\left(z^{\prime}\right) d z^{\prime}$.

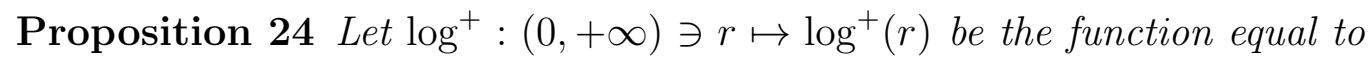
0 for $r \geq 1$ and to $-\log r$ for $r \in(0,1)$. For every $R \geq 1$, set

$$
h_{R}(z)=1_{\{|z| \leq R\}} \sum_{(i, j) \in \Delta_{N}} \log ^{+}\left|z^{i}-z^{j}\right|, \quad z \in \mathbb{R}^{2 N d} .
$$

Then, for any $R_{0}, R, T>0$ there exists a constant $C$ such that, for any $\varepsilon>0$,

$$
\int_{B_{2 N d}\left(0, R_{0}\right)} \mathbb{E}\left[\sup _{t \in[0, T]} h_{R}\left(\varphi_{t}^{\varepsilon}(z)\right)\right] d z \leq C .
$$

Proof. Step 1. For a smooth function $\phi: \mathbb{R} \rightarrow[0,1]$, with support included in $(0,1)$ and with $\int_{0}^{1} \phi(r) d r \leq 2$, let $\log _{\phi}^{+}: \mathbb{R}_{+} \rightarrow \mathbb{R}_{+}$be the smooth function:

$$
\log _{\phi}^{+}(r)=\int_{r}^{1} \frac{\phi(s)}{s} d s \quad \text { for } r \geq 0, \quad \text { so that } \quad\left|\frac{d}{d r} \log _{\phi}^{+}(r)\right| \leq \frac{1}{r} \text { for } r>0 .
$$

As $\phi$ increases towards the indicator function of the interval $(0,1), \log _{\phi}^{+}(r)$ increases towards $\log ^{+}(r)$. Given the function $\mathbb{R}^{2 d} \ni \tilde{z} \mapsto \log _{\phi}^{+}(|\tilde{z}|)$, we have

$$
\begin{aligned}
& \left|\nabla\left[\log _{\phi}^{+}(|\tilde{z}|)\right]\right| \leq C \frac{\mathbf{1}_{\{|\tilde{z}| \leq 1\}}}{|\tilde{z}|}, \\
& \left|\nabla^{2}\left[\log _{\phi}^{+}(|\tilde{z}|)\right]\right| \leq \frac{C}{|\tilde{z}|^{2}}\left(1+\left|\phi^{\prime}\right|(|\tilde{z}|)\right) \cdot \mathbf{1}_{\{|\tilde{z}| \leq 1\}}
\end{aligned}
$$


for a constant $C$ that is independent of the details of $\phi$.

Given $R>0$, let $\theta_{R}: \mathbb{R}^{2 N d} \rightarrow[0,1]$ be a smooth function equal to 1 on $B_{2 N d}(0, R)$, equal to 0 outside $B_{2 N d}(0, R+2)$, with values in $[0,1]$ and with $\sup _{z \in \mathbb{R}^{2 N d}}\left|\nabla \theta_{R}(z)\right| \leq 1$ and $\sup _{z \in \mathbb{R}^{2 N d}}\left|\nabla^{2} \theta_{R}(z)\right| \leq 1$. Define

$$
h_{\phi}^{\theta_{R}}(z)=\theta_{R}(z) \sum_{(i, j) \in \Delta_{N}} \log _{\phi}^{+}\left(\left|z^{i}-z^{j}\right|\right), \quad z \in \mathbb{R}^{2 N d} .
$$

Below, we prove that, given $R_{0}, R>0$, there exists a constant $C$, independent of $\varepsilon$ and the details of $\phi$ and $\theta_{R}$ in $B_{2 N d}(0, R+2) \backslash B_{2 N d}(0, R)$, such that

$$
\mathbb{E} \int_{B\left(0, R_{0}\right)} \sup _{t \in[0, T]} h_{\phi}^{\theta_{R}}\left(\varphi_{t}^{\varepsilon}(z)\right) d z \leq C
$$

Letting $\phi$ increase towards $\mathbf{1}_{(0,1)}$, (41) follows by monotonous convergence.

Step 2. We now prove (44). In the whole argument, we use the compact formulation (14). With the notation $g(\tilde{z})=\log _{\phi}^{+}(|\tilde{z}|)$ and for a generic solution $\left(Z_{t}^{\varepsilon}\right)_{t \geq 0}$ to the smoothed system, we have:

$$
d\left(\theta_{R}\left(Z_{t}^{\varepsilon}\right) g\left(Z_{t}^{i, \varepsilon}-Z_{t}^{j, \varepsilon}\right)\right)=d I_{t}^{1}+d I_{t}^{2}+d I_{t}^{3}, \quad\left\{\begin{array}{l}
d I_{t}^{1}=d I_{t}^{11}+d I_{t}^{12} \\
d I_{t}^{2}=d I_{t}^{21}+d I_{t}^{22}
\end{array}\right.
$$

where

$$
\begin{aligned}
& d I_{t}^{11}=\theta_{R}\left(Z_{t}^{\varepsilon}\right)\left\langle\nabla g\left(Z_{t}^{i, \varepsilon}-Z_{t}^{j, \varepsilon}\right), d\left(Z_{t}^{i, \varepsilon}-Z_{t}^{j, \varepsilon}\right)\right\rangle_{\mathbb{R}^{2 d}}, \\
& d I_{t}^{12}=\frac{\theta_{R}\left(Z_{t}^{\varepsilon}\right)}{2} \sum_{\alpha, \beta=1}^{2 d} \frac{\partial^{2} g}{\partial \tilde{z}_{\alpha} \partial \tilde{z}_{\beta}}\left(Z_{t}^{i, \varepsilon}-Z_{t}^{j, \varepsilon}\right) d\left[\left(Z_{t}^{i, \varepsilon}-Z_{t}^{j, \varepsilon}\right)_{\alpha},\left(Z_{t}^{i, \varepsilon}-Z_{t}^{j, \varepsilon}\right)_{\beta}\right]_{t}, \\
& d I_{t}^{21}=g\left(Z_{t}^{i, \varepsilon}-Z_{t}^{j, \varepsilon}\right)\left\langle\nabla \theta_{R}\left(Z_{t}^{\varepsilon}\right), d Z_{t}^{\varepsilon}\right\rangle_{\mathbb{R}^{2 N d}}, \\
& d I_{t}^{22}=\frac{g\left(Z_{t}^{i, \varepsilon}-Z_{t}^{j, \varepsilon}\right)}{2} \sum_{i^{\prime}, j^{\prime}=1}^{N} \sum_{\alpha, \beta=1}^{2 d} \frac{\partial^{2} \theta_{R}}{\partial\left(z^{i^{\prime}}\right)_{\alpha} \partial\left(z^{j^{\prime}}\right)_{\beta}}\left(Z_{t}^{\varepsilon}\right) d\left[\left(Z^{i^{\prime}, \varepsilon}\right)_{\alpha},\left(Z^{j^{\prime}, \varepsilon}\right)_{\beta}\right]_{t}, \\
& d I_{t}^{3}=\sum_{k=1}^{\infty}\left\langle\nabla g\left(Z_{t}^{i, \varepsilon}-Z_{t}^{j, \varepsilon}\right),\left[\mathbb{A}_{k}^{i}-\mathbb{A}_{k}^{j}\right]\left(Z_{t}^{\varepsilon}\right)\right\rangle_{\mathbb{R}^{2 d}}\left\langle\nabla \theta_{R}\left(Z_{t}^{\varepsilon}\right), \mathbb{A}_{k}\left(Z_{t}^{\varepsilon}\right)\right\rangle_{\mathbb{R}^{2 N d}} d t .
\end{aligned}
$$

We first tackle the mutual variations. By the identity $\left[\mathbb{A}_{k}^{i}-\mathbb{A}_{k}^{j}\right]\left(Z_{t}^{\varepsilon}\right)=$ $\sigma_{k}\left(X_{t}^{i, \varepsilon}\right)-\sigma_{k}\left(X_{t}^{j, \varepsilon}\right)$ and by (42), (12) and (A.3),

$$
\left|d I_{t}^{3}\right| \leq C\left|\nabla \theta_{R}\left(Z_{t}^{\varepsilon}\right)\right| d t
$$


In order to deal with the term $I_{t}^{12}$ we need to analyze the mutual variation $\left[\left(Z^{i, \varepsilon}-Z^{j, \varepsilon}\right)_{\alpha},\left(Z^{i, \varepsilon}-Z^{j, \varepsilon}\right)_{\beta}\right]_{t}$. Obviously, it holds $\left[\left(X^{i, \varepsilon}-X^{j, \varepsilon}\right)_{p},\left(Z^{i, \varepsilon}-\right.\right.$ $\left.\left.Z^{j, \varepsilon}\right)_{\beta}\right]_{t}=0$ for all $p=1, \ldots, d$ and $\beta=1, \ldots, 2 d$, since $X^{i, \varepsilon}-X^{j, \varepsilon}$ is of bounded variation. Moreover, by (12), we have, for $p, q=1, \ldots, d$,

$$
\left|d\left[\left(V_{t}^{i, \varepsilon}-V_{t}^{j, \varepsilon}\right)_{p},\left(V_{t}^{i, \varepsilon}-V_{t}^{j, \varepsilon}\right)_{q}\right]_{t}\right| \leq C\left|X_{t}^{i, \varepsilon}-X_{t}^{j, \varepsilon}\right|^{2} d t
$$

By inequality (43), we get (renaming the constant $C$ )

$$
\begin{aligned}
\left|d I_{t}^{12}\right| & \leq \frac{C \theta_{R}\left(Z_{t}^{\varepsilon}\right)\left(1+\left|\phi^{\prime}\right|\left(\left|Z_{t}^{i, \varepsilon}-Z_{t}^{j, \varepsilon}\right|\right)\right)}{\left|Z_{t}^{i, \varepsilon}-Z_{t}^{j, \varepsilon}\right|^{2}}\left|X_{t}^{i, \varepsilon}-X_{t}^{j, \varepsilon}\right|^{2} d t \\
& \leq C \theta_{R}\left(Z_{t}^{\varepsilon}\right)\left(1+\left|\phi^{\prime}\right|\left(\left|Z_{t}^{i, \varepsilon}-Z_{t}^{j, \varepsilon}\right|\right)\right) d t .
\end{aligned}
$$

Finally, let us deal with $I_{t}^{22}$. As above, the only terms to be non-zero in the variation $d\left[\left(Z^{i^{\prime}, \varepsilon}\right)_{\alpha},\left(Z^{j^{\prime}, \varepsilon}\right)_{\beta}\right]_{t}$ are the terms $d\left[\left(V_{t}^{i^{\prime}, \varepsilon}\right)_{p},\left(V_{t}^{j^{\prime}, \varepsilon}\right)_{q}\right]_{t}$. By boundedness of $|Q(\tilde{x}, \tilde{x})|$, we claim $\left|d\left[\left(V_{t}^{i^{\prime}, \varepsilon}\right)_{p},\left(V_{t}^{j^{\prime}, \varepsilon}\right)_{q}\right]_{t}\right| \leq C d t$ and thus

$$
\left|d I_{t}^{22}\right| \leq C g\left(Z_{t}^{i, \varepsilon}-Z_{t}^{j, \varepsilon}\right)\left|\nabla^{2} \theta_{R}\left(Z_{t}^{\varepsilon}\right)\right| d t .
$$

Step 3. Split now $d I_{t}^{11}$ and $d I_{t}^{21}$ into $d I_{t}^{111}+d I_{t}^{112}$ and $d I_{t}^{211}+d I_{t}^{212}$, where

$$
\begin{aligned}
& d I_{t}^{111}=\theta_{R}\left(Z_{t}^{\varepsilon}\right)\left\langle\nabla g\left(Z_{t}^{i, \varepsilon}-Z_{t}^{j, \varepsilon}\right), \mathbb{F}_{\varepsilon}^{i}\left(Z_{t}^{\varepsilon}\right)-\mathbb{F}_{\varepsilon}^{j}\left(Z_{t}^{\varepsilon}\right)\right\rangle_{\mathbb{R}^{2 d}} d t \\
& d I_{t}^{112}=\theta_{R}\left(Z_{t}^{\varepsilon}\right) \sum_{k=1}^{\infty}\left\langle\nabla g\left(Z_{t}^{i, \varepsilon}-Z_{t}^{j, \varepsilon}\right), \mathbb{A}_{k}^{i}\left(Z_{t}^{\varepsilon}\right)-\mathbb{A}_{k}^{j}\left(Z_{t}^{\varepsilon}\right)\right\rangle_{\mathbb{R}^{2 d}} d W_{t}^{k}, \\
& d I_{t}^{211}=g\left(Z_{t}^{i, \varepsilon}-Z_{t}^{j, \varepsilon}\right)\left\langle\nabla \theta_{R}\left(Z_{t}^{\varepsilon}\right), \mathbb{F}_{\varepsilon}\left(Z_{t}^{\varepsilon}\right)\right\rangle_{\mathbb{R}^{2 N d}} d t \\
& d I_{t}^{212}=g\left(Z_{t}^{i, \varepsilon}-Z_{t}^{j, \varepsilon}\right) \sum_{k=1}^{\infty}\left\langle\nabla \theta_{R}\left(Z_{t}^{\varepsilon}\right), \mathbb{A}_{k}\left(Z_{t}^{\varepsilon}\right)\right\rangle_{\mathbb{R}^{2 N d}} d W_{t}^{k}
\end{aligned}
$$

By (42) and by boundedness of $\mathbb{F}_{\varepsilon}$ on $B_{2 N d}(0, R)$, we have

$$
d I_{t}^{111} \leq C \frac{\theta_{R}\left(Z_{t}^{\varepsilon}\right)}{\left|Z_{t}^{i, \varepsilon}-Z_{t}^{j, \varepsilon}\right|} d t, \quad d I_{t}^{211} \leq C g\left(Z_{t}^{i, \varepsilon}-Z_{t}^{j, \varepsilon}\right)\left|\nabla \theta_{R}\left(Z_{t}^{\varepsilon}\right)\right| d t .
$$

Step 4. We now deal with the martingale terms $I^{112}$ and $I^{212}$. By (42), (12), by boundedness of $|Q(\tilde{x}, \tilde{x})|$ and Doob's inequality,

$$
\begin{aligned}
& \mathbb{E}\left[\sup _{t \in[0, T]}\left|I_{T}^{112}\right|^{2}\right] \leq C \mathbb{E}\left[\int_{0}^{T} \theta_{R}^{2}\left(Z_{s}^{\varepsilon}\right) d s\right], \\
& \mathbb{E}\left[\sup _{t \in[0, T]}\left|I_{T}^{212}\right|^{2}\right] \leq C \mathbb{E}\left[\int_{0}^{T} g^{2}\left(Z_{s}^{i, \varepsilon}-Z_{s}^{j, \varepsilon}\right)\left|\nabla \theta_{R}\left(Z_{s}^{\varepsilon}\right)\right|^{2} d s\right] .
\end{aligned}
$$


From the above bounds, together with (45), (46), (47), (48) and (49), and making use of the following estimates

$$
\begin{aligned}
& \max \left(\theta_{R}(z),\left|\nabla_{R} \theta(z)\right|,\left|\nabla_{R}^{2} \theta(z)\right|\right) \leq \mathbf{1}_{\{|z| \leq R+2\}}, \quad z \in \mathbb{R}^{2 N d}, \\
& \max \left(g(\tilde{z}), g^{2}(\tilde{z})\right) \leq \frac{C}{|\tilde{z}|}, \quad \tilde{z} \in \mathbb{R}^{2 d} .
\end{aligned}
$$

we deduce (with $Z_{0}^{\varepsilon}=z$ )

$$
\begin{aligned}
& \mathbb{E}\left[\sup _{t \in[0, T]}\left(\theta_{R}\left(Z_{t}^{\varepsilon}\right) \log _{\phi}^{+}\left(\left|Z_{t}^{i, \varepsilon}-Z_{t}^{j, \varepsilon}\right|\right)\right)\right] \leq \theta_{R}(z) \log _{\phi}^{+}\left(\left|z^{i}-z^{j}\right|\right) \\
& \quad+C\left(1+\mathbb{E} \int_{0}^{T} \mathbf{1}_{\left\{\left|Z_{s}^{\varepsilon}\right| \leq R+2\right\}}\left(\frac{1}{\left|Z_{s}^{i, \varepsilon}-Z_{s}^{j, \varepsilon}\right|}+\left|\phi^{\prime}\right|\left(\left|Z_{s}^{i, \varepsilon}-Z_{s}^{j, \varepsilon}\right|\right)\right) d s\right) .
\end{aligned}
$$

Step 5. We now integrate on a ball $B_{2 N d}\left(0, R_{0}\right)$ of $\mathbb{R}^{2 N d}$ with respect to the initial conditions. Applying Lemma 23, we get

$$
\begin{aligned}
& \int_{B_{2 N d}\left(0, R_{0}\right)} \mathbb{E}\left[\sup _{t \in[0, T]}\left(\theta_{R}\left(\varphi_{t}^{\varepsilon}(z)\right) \log _{\phi}^{+}\left(\left|\varphi_{t}^{i, \varepsilon}(z)-\varphi_{t}^{j, \varepsilon}(z)\right|\right)\right)\right] d z \\
& \leq \int_{B_{2 N d}\left(0, R_{0}\right)} \theta_{R}(z) \log _{\phi}^{+}\left(\left|z^{i}-z^{j}\right|\right) d z \\
& +C\left[R_{0}^{2 N d}+\mathbb{E} \int_{0}^{T} \int_{\{|z| \leq R+2\}}\left(\frac{1}{\left|z^{i}-z^{j}\right|}+\left|\phi^{\prime}\right|\left(\left|z^{i}-z^{j}\right|\right)\right) d z d s\right] .
\end{aligned}
$$

By a spherical change of variable, the integral of $\left|\phi^{\prime}\right|\left(\left|z^{i}-z^{j}\right|\right)$ by $C \int_{0}^{1}\left|\phi^{\prime}(r)\right| d r$, which is less than $2 C$. This completes the proof.

Lemma 25 Given $R_{0}, T>0$, define $\mathfrak{m}$ as the normalized product measure $\mathcal{V}_{2 N d}^{-1}\left(R_{0}\right) \cdot \mathrm{Leb}_{2 N d} \otimes \mathbb{P}$ on $\mathfrak{O}=B_{2 N d}\left(0, R_{0}\right) \times \Omega$. Then,

$$
\limsup _{\epsilon \rightarrow 0} \mathfrak{m}\left\{\inf _{\varepsilon>0} \inf _{t \in[0, T]}\left|\varphi_{(i, j) \in \Delta_{N}}^{i, \varepsilon}(z, \omega)-\varphi_{t}^{j, \varepsilon}(z, \omega)\right| \leq \epsilon\right\}=0 .
$$

(For a measurable function $\phi: \mathfrak{O} \rightarrow \mathbb{R}$ w.r.t. the product $\sigma$-field on $\mathfrak{O}$ and a Borel set $A \subset \mathbb{R}, \mathfrak{m}\{\phi(z, \omega) \in A\}$ stands for $\mathfrak{m}\{(z, \omega) \in \mathfrak{O}: \phi(z, \omega) \in A\}$.)

Proof. By boundedness of $F_{\varepsilon}$ and $Q(\tilde{x}, \tilde{x})$ and by Markov inequality, it is well-seen that, for any $R_{0}>0$, there exists a constant $C$, only depending on 
$R_{0}$ and $T$, such that, for any $R>0, \mathfrak{m}\left\{\sup _{t \in[0, T]}\left|\varphi_{t}^{\varepsilon}(z, \omega)\right|>R\right\} \leq C_{R_{0}} / R$. Moreover, by Proposition 24,

$$
\mathfrak{m}\left\{\sup _{t \in[0, T]}\left[\mathbf{1}_{\left\{\left|\varphi_{t}^{\varepsilon}(z, \omega)\right| \leq R\right\}} \sum_{(i, j) \in \Delta_{N}} \log ^{+}\left|\varphi_{t}^{i, \varepsilon}(z, \omega)-\varphi_{t}^{j, \varepsilon}(z, \omega)\right|\right]>K\right\} \leq \frac{C}{K},
$$

$C$ possibly depending upon $R$ as well. The proof is easily completed.

Lemma 26 Given $R_{0}, T>0$, keep the same definition for $\mathfrak{m}$ as above. Then,

$$
\begin{aligned}
& \lim _{(\epsilon, A) \rightarrow(0,+\infty)} \sup _{\varepsilon>0} \sup _{0<\delta_{0}<1} \mathfrak{m}\{(z, \omega) \in \mathfrak{O}: \\
& \left.\operatorname{Leb}_{1}\left(t \in[0, T]: \inf _{(i, j) \in \Delta_{N}}\left|\tilde{\pi}_{x}\left[\varphi_{t}^{i, \varepsilon}(z, \omega)-\varphi_{t}^{j, \varepsilon}(z, \omega)\right]\right| \leq \frac{\delta_{0} \epsilon}{A}\right)>A \delta_{0}\right\}=0 .
\end{aligned}
$$

Proof. The proof follows from Lemma 25 and Proposition 27 below. Indeed, by boundedness of $F_{\varepsilon}$ and $Q(\tilde{x}, \tilde{x})$, the probability that the $v$-coordinate of $\left(\varphi_{t}^{\varepsilon}(z)\right)_{0 \leq t \leq T}$ is $1 / 4$-Hölder continuous with $A$ as Hölder constant converges towards 1 as $A$ tends to $+\infty$, uniformly in $\varepsilon>0$ and in $z \in B_{2 N d}\left(0, R_{0}\right)$.

Proposition 27 Given $A, R_{0}, T>0$, let $\left(\zeta_{t}=\left(\chi_{t}, \nu_{t}\right)\right)_{0 \leq t \leq T}$ be a continuous path with values in $\mathbb{R}^{2 N d}$ such that $\zeta_{0}=z \in B_{2 N d}\left(0, R_{0}\right),\left(\nu_{t}^{i}\right)_{t>0}$ is a $1 / 4$ Hölder continuous $\mathbb{R}^{d}$-valued path with $A$ as Hölder constant, for $1 \leq i \leq N$, and $\left[d \chi_{t}^{i} / d t\right]=\nu_{t}^{i}$, for $t \in[0, T]$ and $i \in\{1, \ldots, N\}$. Then, there exists a constant $C$, depending on $d, A, N, R_{0}$ and $T$ only, such that, for any $\epsilon, \delta_{0} \in(0,1), \inf _{t \in[0, T]} \inf _{(i, j) \in \Delta_{N}}\left|\zeta_{t}^{i}-\zeta_{t}^{j}\right| \geq \epsilon$ implies

$$
\operatorname{Leb}_{1}\left(t \in[0, T]: \inf _{(i, j) \in \Delta_{N}}\left|\chi_{t}^{i}-\chi_{t}^{j}\right| \leq \delta_{0} \frac{\epsilon^{5}}{C}\right) \leq C \delta_{0} .
$$

Proof. Assume that there exist $\delta \in(0, \epsilon), t_{0} \in[0, T)$ and $(i, j) \in \Delta_{N}$ such that $\left|\chi_{t_{0}}^{i}-\chi_{t_{0}}^{j}\right| \leq \delta$. Since $\inf _{t \in[0, T]} \inf _{(i, j) \in \Delta_{N}}\left|\zeta_{t}^{i}-\zeta_{t}^{j}\right| \geq \epsilon$, we deduce $\left|\nu_{t_{0}}^{i}-\nu_{t_{0}}^{j}\right| \geq \sqrt{\epsilon^{2}-\delta^{2}}$. By Hölder property of $\left(\nu_{t}\right)_{0 \leq t \leq T}$, there exists a constant $C$, independent of $\epsilon, t_{0}$ and $\delta$, such that

$$
\left|\nu_{t}^{i}-\nu_{t}^{j}\right| \geq \sqrt{\epsilon^{2}-\delta^{2}}-C\left(t-t_{0}\right)^{1 / 4}, \quad t_{0} \leq t \leq T .
$$

Therefore, there exists one coordinate $\ell \in\{1, \ldots, d\}$ such that

$$
\left|\left(\nu_{t}^{i}-\nu_{t}^{j}\right)_{\ell}\right| \geq \frac{\sqrt{\epsilon^{2}-\delta^{2}}-C\left(t-t_{0}\right)^{1 / 4}}{\sqrt{d}}, \quad t_{0} \leq t \leq T .
$$


For $C\left(t-t_{0}\right)^{1 / 4}<\sqrt{\epsilon^{2}-\delta^{2}}$, the right-hand side is always positive so that $\left(\nu_{t}^{i}-\nu_{t}^{j}\right)_{\ell}$ cannot vanish. By continuity, it is of constant sign. Therefore,

$$
\left|\chi_{t}^{i}-\chi_{t}^{j}\right| \geq\left|\left(\chi_{t}^{i}-\chi_{t}^{j}\right)_{\ell}\right| \geq\left(t-t_{0}\right) \frac{\sqrt{\epsilon^{2}-\delta^{2}}-C\left(t-t_{0}\right)^{1 / 4}}{\sqrt{d}}-\delta,
$$

for $C\left(t-t_{0}\right)^{1 / 4} \leq \sqrt{\epsilon^{2}-\delta^{2}}$. For $\delta \leq \epsilon / 2$, we deduce $\left|\chi_{t}^{i}-\chi_{t}^{j}\right| \geq \epsilon(t-$ $\left.t_{0}\right) /(4 \sqrt{d})-\delta$, for $C\left(t-t_{0}\right)^{1 / 4} \leq \epsilon / 4$. Finally, for $8 \sqrt{d} \delta / \epsilon \leq t-t_{0} \leq \epsilon^{4} /(4 C)^{4}$, $\left|\chi_{t}^{i}-\chi_{t}^{j}\right| \geq \delta$. Modifying $C$ if necessary, we deduce that

$$
\left|\chi_{t}^{i}-\chi_{t}^{j}\right| \geq \delta
$$

for $C \delta / \epsilon \leq t-t_{0} \leq \epsilon^{4} / C$ and $\delta \leq \epsilon / 2$. Assume now w.l.o.g. that $C \geq 2$ and choose $\delta$ of the form $\delta_{0} \epsilon^{5} / C^{2}$ with $\delta_{0} \leq 1$. Define the set

$$
\mathcal{I}_{x}\left(\delta_{0}, \epsilon\right)=\left\{t \in[0, T]:\left|\chi_{t}^{i}-\chi_{t}^{j}\right| \leq \delta_{0} \epsilon^{5} / C^{2}\right\}
$$

By $(50), t_{0} \in \mathcal{I}_{x}\left(\delta_{0}, \epsilon\right) \Rightarrow\left[t_{0}+\delta_{0} \epsilon^{4} / C, t_{0}+\epsilon^{4} / C\right] \cap \mathcal{I}_{x}\left(\delta_{0}, \epsilon\right)=\emptyset$. Therefore,

$$
\operatorname{Leb}_{1}\left(\mathcal{I}_{x}\left(\delta_{0}, \epsilon\right)\right) \leq \delta_{0} \epsilon^{4} / C\left\lceil T C / \epsilon^{4}\right\rceil \leq \delta_{0}(T+1) .
$$

\subsection{No coalescence for a.e. initial configuration}

As a consequence of the previous estimates, we prove (the result below might be compared with [8] and [1] about a.e. solvability of ODEs, but therein uniqueness is investigated through uniqueness of a regular Lagrangian flow):

Theorem 28 Under Assumptions (A.1-3), for Lebesgue almost every z, equation (13) has one and only one global strong solution.

Proof. Step 1. We here consider $\Xi=\mathcal{C}\left([0,+\infty), \mathbb{R}^{2 N d}\right) \otimes \mathcal{C}([0,+\infty), \mathbb{R})^{\otimes \mathbb{N} \backslash\{0\}}$ endowed with the product $\sigma$-field $\mathcal{X}$ of the Borel $\sigma$-fields. For $R_{0}>0$ and $\varepsilon>0$ and with the same notations as in Lemma 25 , we endow the pair $(\Xi, \mathcal{X})$ with the probability $\mathbb{Q}^{\varepsilon}$ defined on the cylinders as

$$
\begin{aligned}
& \mathbb{Q}^{\varepsilon}\left(A_{0} \times A_{1} \times \cdots \times A_{k} \times \mathcal{C}([0,+\infty), \mathbb{R}) \times \cdots\right) \\
& =\mathfrak{m}\left\{\left(\varphi_{t}^{\varepsilon}(z)\right)_{t \geq 0} \in A_{0},\left(W_{t}^{1}, \ldots, W_{t}^{k}\right) \in A_{1} \times \cdots \times A_{k}\right\},
\end{aligned}
$$

where $A_{0}$ is a Borel subset of $\mathcal{C}\left([0,+\infty), \mathbb{R}^{2 N d}\right)$ and $A_{1}, \ldots, A_{N}$ are Borel subsets of $\mathcal{C}([0,+\infty), \mathbb{R})$. The $\sigma$-field $\mathcal{X}$ coincides with the Borel $\sigma$-field generated by the standard product metric on the product space $\Xi$. In particular, 
the notion of tightness is relevant for probability measures on the pair $(\Xi, \mathcal{X})$ : it is well-checked that the family $\left(\mathbb{Q}^{\varepsilon}\right)_{\varepsilon>0}$ is tight.

Denoting by $\mathbb{Q}$ the limit of some convergent sequence $\left(\mathbb{Q}^{\varepsilon_{n}}\right)_{n \in \mathbb{N}}$ for a decreasing sequence of positive reals $\left(\varepsilon_{n}\right)_{n \in \mathbb{N}}$ converging towards 0 , we investigate the properties of the canonical process under $\mathbb{Q}$, denoted by $\xi_{t}=\left(\xi_{t}^{k}\right)_{k \in \mathbb{N}}$,

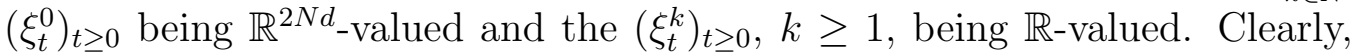
the family $\left(\left(\xi_{t}^{k}\right)_{t \geq 0}\right)_{k \in \mathbb{N} \backslash\{0\}}$ is a family of independent Brownian motions under $\mathbb{Q}$. Moreover, the marginal law of $\Xi \ni \xi \mapsto \xi_{0}^{0}$ is the uniform distribution on the ball $B_{2 N d}\left(0, R_{0}\right)$.

For any $\epsilon>0$, the set $\left\{\xi \in \Xi: \inf _{t \in[0, T]} \inf _{(i, j) \in \Delta_{N}}\left|\pi_{i}\left(\xi_{t}^{0}\right)-\pi_{j}\left(\xi_{t}^{0}\right)\right|<\epsilon\right\}$ is open in $\Xi$. Using the porte-manteau Theorem to pass to the limit in Lemma 25 and letting $\epsilon$ tend to 0 , we deduce that, for any $T>0$,

$$
\mathbb{Q}\left\{\xi \in \Xi: \inf _{(i, j) \in \Delta_{N}} \inf _{t \in[0, T]}\left|\pi_{i}\left(\xi_{t}^{0}\right)-\pi_{j}\left(\xi_{t}^{0}\right)\right|=0\right\}=0
$$

Similarly, the set $\left\{\xi \in \Xi: \operatorname{Leb}_{1}\left(t \in[0, T]: \inf _{(i, j) \in \Delta_{N}}\left|\pi_{i, x}\left(\xi_{t}^{0}\right)-\pi_{j, x}\left(\xi_{t}^{0}\right)\right|<\right.\right.$ $\left.\left.\delta_{0} \epsilon / A\right)>A \delta_{0}\right\}$ is open in $\Xi$. Using the porte-manteau Theorem to pass to the limit in Lemma 26 and letting $\delta_{0}$ tend to 0 first and then $(\epsilon, A)$ to $(0,+\infty)$, we obtain

$$
\mathbb{Q}\left\{\xi \in \Xi: \operatorname{Leb}_{1}\left(t \in[0, T]: \inf _{(i, j) \in \Delta_{N}}\left|\pi_{i, x}\left(\xi_{t}^{0}\right)-\pi_{j, x}\left(\xi_{t}^{0}\right)\right|=0\right)>0\right\}=0 .
$$

Set now $\xi_{t}^{0}=\left(\chi_{t}^{0}, \nu_{t}^{0}\right)$, with $\chi_{t}^{0}=\Pi_{x}\left(\xi_{t}^{0}\right)$ and $\nu_{t}^{0}=\Pi_{v}\left(\xi_{t}^{0}\right), t \geq 0$. Set also

$$
\tilde{\nu}_{t}^{0}=\nu_{t}^{0}-\int_{0}^{t} \Pi_{v}\left(\mathbb{F}\left(\chi_{s}^{0}\right)\right) d s, \quad t \geq 0 .
$$

We claim that $\left(\tilde{\nu}_{t}^{0}\right)_{t \geq 0}$ is a square-integrable continuous martingale under $\mathbb{Q}$ w.r.t. the filtration $\left(\mathcal{G}_{t}^{0}=\sigma\left(\xi_{s}^{k}, s \leq t, k \in \mathbb{N}\right)\right)_{t \geq 0}$ with the mutual variations

$$
\begin{aligned}
& {\left[\left(\tilde{\nu}^{0}\right)^{i},\left(\tilde{\nu}^{0}\right)^{j}\right]_{t}=\int_{0}^{t} Q\left(\left(\chi_{s}^{0}\right)^{i},\left(\chi_{s}^{0}\right)^{j}\right) d s, \quad i, j \in\{1, \ldots, d\},} \\
& {\left[\left(\tilde{\nu}^{0}\right)^{i}, \xi^{k}\right]_{t}=\int_{0}^{t} \sigma_{k}\left(\left(\chi_{s}^{0}\right)^{i}\right) d s, \quad i \in\{1, \ldots, d\}, k \in \mathbb{N} \backslash\{0\} .}
\end{aligned}
$$

The proof is quite standard and consists in passing to the limit in the martingale properties characterizing the dynamics of $\left(Z_{t}^{\varepsilon}\right)_{t \geq 0}$. The only difficulty 
is to pass to the limit along the mollified drifts. For $T>0$, we thus prove that

$$
\left(\int_{0}^{t} \mathbb{F}_{\varepsilon}\left(\xi_{s}^{0}\right) d s\right)_{0 \leq t \leq T} \cdot \mathbb{Q}^{\varepsilon} \underset{\varepsilon \rightarrow 0}{\Rightarrow}\left(\int_{0}^{t} \mathbb{F}\left(\xi_{s}^{0}\right) d s\right)_{0 \leq t \leq T} \cdot \mathbb{Q}
$$

where the left- and the right-hand sides indicate the distributions of the indicated processes under the indicated measures on $\mathcal{C}\left([0, T], \mathbb{R}^{2 N d}\right)$ and $\Rightarrow$ stands for the convergence in distribution. By boundedness of $F_{\varepsilon}$, we emphasize that there exists a constant $C>0$, independent of $\varepsilon$ such that, for any $a>0$ and any $\varepsilon^{\prime}>\varepsilon$,

$$
\begin{aligned}
& \mathbb{Q}^{\varepsilon}\left(\sup _{0 \leq t \leq T}\left|\int_{0}^{t} \mathbb{F}_{\varepsilon}\left(\xi_{s}^{0}\right) d s-\int_{0}^{t} \mathbb{F}\left(\xi_{s}^{0}\right) d s\right|>a\right) \\
& \leq \mathbb{Q}^{\varepsilon}\left(C \operatorname{Leb}_{1}\left(t \in[0, T]: \inf _{(i, j) \in \Delta_{N}}\left|\pi_{i, x}\left(\xi_{t}^{0}\right)-\pi_{j, x}\left(\xi_{t}^{0}\right)\right| \leq \varepsilon^{\prime}\right) \geq a\right) .
\end{aligned}
$$

The event in the right-hand side is closed in $\Xi$, so that

$$
\begin{aligned}
& \limsup _{\varepsilon \rightarrow 0} \mathbb{Q}^{\varepsilon}\left(\sup _{0 \leq t \leq T}\left|\int_{0}^{t} \mathbb{F}_{\varepsilon}\left(\xi_{s}^{0}\right) d s-\int_{0}^{t} \mathbb{F}\left(\xi_{s}^{0}\right) d s\right|>a\right) \\
& \leq \mathbb{Q}\left(C \operatorname{Leb}_{1}\left(t \in[0, T]: \inf _{(i, j) \in \Delta_{N}}\left|\pi_{i, x}\left(\xi_{t}^{0}\right)-\pi_{j, x}\left(\xi_{t}^{0}\right)\right| \leq \varepsilon^{\prime}\right) \geq a\right) .
\end{aligned}
$$

Letting $\varepsilon^{\prime}$ tend to 0 in (57), we deduce from (52) that the left-hand side is 0 . Therefore, to prove (56), it is sufficient to prove

$$
\left(\int_{0}^{t} \mathbb{F}\left(\xi_{s}\right) d s\right)_{0 \leq t \leq T} \cdot \mathbb{Q}^{\varepsilon} \underset{\varepsilon \rightarrow 0}{\Rightarrow}\left(\int_{0}^{t} \mathbb{F}\left(\xi_{s}\right) d s\right)_{0 \leq t \leq T} \cdot \mathbb{Q} .
$$

By dominated convergence Theorem, the map $\mathcal{C}\left([0, T], \mathbb{R}^{2 N d}\right) \ni\left(\xi_{t}\right)_{0 \leq t \leq T} \mapsto$ $\left(\int_{0}^{t} \mathbb{F}\left(\xi_{s}\right) d s\right)_{0 \leq t \leq T} \in \mathcal{C}\left([0, T], \mathbb{R}^{2 N d}\right)$ is continuous at any path $\xi$ for which $\operatorname{Leb}_{1}\left(t \in[0, T]: \inf _{(i, j) \in \Delta_{N}}\left|\pi_{i, x}\left(\xi_{t}\right)-\pi_{j, x}\left(\xi_{t}\right)\right|=0\right)=0$. By (52) again, this is true a.s. under $\mathbb{Q}$ : by continuous mapping Theorem, we complete the proof of (56). Thus, $\left(\tilde{\nu}^{0}\right)_{t \geq 0}$ in (53) satisfies the announced martingale propery.

Step 2. Denote by $\left(\mathcal{G}_{t}\right)_{t \geq 0}$ the right-continuous version of $\left(\mathcal{G}_{t}\right)_{t \geq 0}$ augmented with $\mathbb{Q}$-null sets. Clearly, $\left(\tilde{\nu}_{t}^{0}\right)_{t \geq 0}$ is a square-integrable continuous martingale under $\mathbb{Q}$ w.r.t. $\left(\mathcal{G}_{t}\right)_{t \geq 0}$ and both (54) and (55) remain true. In particular, we can compute

$$
\left[\tilde{\nu}^{0}-\sum_{k \in \mathbb{N} \backslash\{0\}} \int_{0}^{\cdot} A_{k}\left(\chi_{s}^{0}\right) d \xi_{s}^{k}\right]_{t}=0, \quad t \geq 0,
$$


so that, $\mathbb{Q}$-a.s.,

$$
\xi_{t}^{0}=\xi_{0}^{0}+\int_{0}^{t} \mathbb{F}\left(\xi_{s}^{0}\right) d s+\sum_{k \in \mathbb{N} \backslash\{0\}} \int_{0}^{t} \mathbb{A}_{k}\left(\chi_{s}^{0}\right) d \xi_{s}^{k}, \quad t \geq 0 .
$$

We denote by $(\mathfrak{Q}(\cdot, z))_{z \in B_{2 N d}\left(0, R_{0}\right)}$ a family of regular conditional probabilities of $\mathbb{Q}$ given the random variable $\Xi \ni \xi \mapsto \xi_{0}^{0}$. It is plain to see that, for a.e. $z \in B_{2 N d}\left(0, R_{0}\right),\left(\left(\xi_{t}^{k}\right)_{t \geq 0}\right)_{k \in \mathbb{N} \backslash\{0\}}$ are independent Brownian motions under $\mathfrak{Q}(\cdot, z),\left(\tilde{\nu}_{t}^{0}\right)_{t \geq 0}$ is a square-integrable continuous martingale under $\mathfrak{Q}(\cdot, z)$ w.r.t. $\left(\mathcal{G}_{t}\right)_{t \geq 0}$ and both $(54)$ and (55) remain true under $\mathfrak{Q}(\cdot, z)$. We deduce that, for a.e. $z \in B_{2 N d}\left(0, R_{0}\right)$, (58) holds true $\mathfrak{Q}(\cdot, z)$-a.s. with $\xi_{0}^{0}=z$ therein. (Notice that, a.e. on $B_{2 N d}\left(0, R_{0}\right)$, the version of the stochastic integral may be chosen independently of $z$. Of course, its distribution under $\mathfrak{Q}(\cdot, z)$ depends on $z$.) From (51) and (52), we deduce that, for a.e. $z \in$ $B_{2 N d}\left(0, R_{0}\right)$, there is no coalesence in the phase space with probability 1 under $\mathfrak{Q}(\cdot, z)$ and that the set of instants where coalescence occurs in the space of positions is of zero Lebesgue measure with probability 1 under $\mathfrak{Q}(\cdot, z)$.

Step 3 We now prove that pathwise uniqueness holds for solutions that remain in $\Gamma_{N}$ almost-surely. We are thus given two solutions $\left(\zeta_{t}\right)_{t \geq 0}$ and $\left(\zeta_{t}^{\prime}\right)_{t \geq 0}$ to (13) with $\zeta_{0}=\zeta_{0}^{\prime}=z \in \Gamma_{N},\left(\zeta_{t}\right)_{t \geq 0}$ being almost-surely free of coalescence in the phase space. (Processes $\left(\zeta_{t}\right)_{t \geq 0}$ and $\left(\zeta_{t}^{\prime}\right)_{t \geq 0}$ are $\mathbb{R}^{2 N d}$-valued and play the same role as $\left(Z_{t}\right)_{t \geq 0}$.) Denoting by $\tau=\inf \left\{t \geq 0: \zeta_{t} \neq \zeta_{t}^{\prime}\right\}$, the point is thus to prove that $\mathbb{P}\{\tau=+\infty\}=1$.

On the set $\{\tau<+\infty\}$ (if not empty), we have $\zeta_{\tau}=\zeta_{\tau}^{\prime} \in \Gamma_{N}$ since $\left(\zeta_{t}\right)_{t \geq 0}$ is free of coalescence in the phase space. Put it differently, we have, $\mathbb{P}$-a.s., $\zeta_{\tau \wedge n}=\zeta_{\tau \wedge n}^{\prime} \in \Gamma_{N}$ for all $n \in \mathbb{N}$. Pathwise uniqueness follows from:

Lemma 29 Let $Z_{0}$ be a random variable with values in $\Gamma_{N}$ and let $\left(\zeta_{t}\right)_{t \geq 0}$ and $\left(\zeta_{t}^{\prime}\right)_{t \geq 0}$ stand for two solutions to (13) with $Z_{0}$ as initial condition, $\left(\zeta_{t}\right)_{t \geq 0}$ being free of coalescence in the phase space. Then, there exists a stopping time $\rho, \mathbb{P}\{\rho>0\}=1$, such that $\left(\zeta_{t}\right)_{t \geq 0}$ and $\left(\zeta_{t}^{\prime}\right)_{t \geq 0}$ match almost-surely on $[0, \rho]$.

Applying Lemma 29 with $Z_{0}=\zeta_{\tau \wedge n}$ as initial conditions, we deduce that $\mathbb{P}\{\tau \geq n\}=1$ for any $n \in \mathbb{N}$, that is $\mathbb{P}\{\tau=+\infty\}=1$, as announced.

We turn to the proof of Lemma 29. We write $Z_{0}=\left(X_{0}, V_{0}\right)$, with $X_{0}=$ $\Pi_{x}\left(Z_{0}\right)$ and $V_{0}=\Pi_{v}\left(Z_{0}\right)$, and $\zeta_{t}=\left(\chi_{t}, \nu_{t}\right)$, with $\chi_{t}=\Pi_{x}\left(\zeta_{t}\right)$ and $\nu_{t}=\Pi_{v}\left(\zeta_{t}\right)$, for $t \geq 0$. On the same model, we write $\zeta_{t}^{\prime}=\left(\chi_{t}^{\prime}, \nu_{t}^{\prime}\right)$ for $t \geq 0$. Setting $\rho^{1, i, j}=\inf \left(\rho_{\zeta}^{1, i, j}, \rho_{\zeta^{\prime}}^{1, i, j}\right)$, with

$$
\rho_{\zeta}^{1, i, j}=\inf \left\{t \geq 0:\left|\chi_{t}^{i}-\chi_{t}^{j}\right| \leq\left|X_{0}^{i}-X_{0}^{j}\right| / 2\right\}
$$


(with a similar definition for $\left.\rho_{\zeta^{\prime}}^{1, i, j}\right)$ and $\rho^{2, i, j}=\inf \left(\rho_{\zeta}^{2, i, j}, \rho_{\zeta^{\prime}}^{2, i, j}\right)$, with

$$
\rho_{\zeta}^{2, i, j}=\inf \left\{t>0:\left|\chi_{t}^{i}-\chi_{t}^{j}\right| \leq t\left|V_{0}^{i}-V_{0}^{j}\right| / 2\right\}
$$

(with the convention that $\rho_{\zeta}^{2, i, j}=0$ if $V_{0}^{i}-V_{0}^{j}=0$, and with a similar definition for $\left.\rho_{\zeta^{\prime}}^{2, i, j}\right)$, we put $\rho^{i, j}=\max \left(\rho^{1, i, j}, \rho^{2, i, j}\right)$.

We first prove that $\rho^{i, j}$ is a.s. positive. If $\rho^{1, i, j}(\omega)$ is zero for a given $\omega \in \Omega$, it holds $\rho_{\zeta}^{1, i, j}(\omega)=0$ or $\rho_{\zeta^{\prime}}^{1, i, j}(\omega)=0$, so that $\left|X_{0}^{i}(\omega)-X_{0}^{j}(\omega)\right|=0$. Since $Z_{0}$ has values in $\Gamma_{N}$, we have $\left|V_{0}^{i}(\omega)-V_{0}^{j}(\omega)\right|>0$. Since the paths of $\left(\zeta_{t}\right)_{t \geq 0}$ and $\left(\zeta_{t}^{\prime}\right)_{t \geq 0}$ are (a.s.) 1/4-Hölder continuous, we also have:

$$
\left|\nu_{t}^{i}(\omega)-\nu_{t}^{j}(\omega)-\left(V_{0}^{i}(\omega)-V_{0}^{j}(\omega)\right)\right| \leq C(\omega) t^{1 / 4}, \quad t \in[0,1],
$$

for a finite constant $C(\omega)$ depending on $\omega$. We deduce that, for any $t \in(0,1]$,

$$
\left|\chi_{t}^{i}(\omega)-\chi_{t}^{j}(\omega)-t\left(V_{0}^{i}(\omega)-V_{0}^{j}(\omega)\right)\right| \leq C(\omega) t^{5 / 4}
$$

Therefore,

$$
\left|\chi_{t}^{i}(\omega)-\chi_{t}^{j}(\omega)\right| \geq \frac{t}{2}\left|V_{0}^{i}(\omega)-V_{0}^{j}(\omega)\right|,
$$

for $C(\omega) t^{1 / 4} \leq(1 / 2)\left|V_{0}^{i}(\omega)-V_{0}^{j}(\omega)\right|$. Therefore, $\rho_{\zeta}^{2, i, j}(\omega)>0$. Similarly, $\rho_{\zeta^{\prime}}^{2, i, j}(\omega)>0$.

On $[0, \rho]$, the drift $F\left(\zeta_{t}^{i}-\zeta_{t}^{j}\right)$ in (13) satisfies

$$
\left|F\left(\chi_{t}^{i}-\chi_{t}^{j}\right)-F\left(\left(\chi_{t}^{\prime}\right)^{i}-\left(\chi_{t}^{\prime}\right)^{j}\right)\right| \leq C t^{-1}\left|\chi_{t}-\chi_{t}^{\prime}\right| \leq C \sup _{0 \leq s \leq t}\left|\nu_{s}-\nu_{s}^{\prime}\right|
$$

the constant $C$ depending upon the randomness through $Z^{0}$ only. Indeed, if $\rho^{1, i, j}>0,\left|X_{0}^{i}-X_{0}^{j}\right|$ must be (strictly) positive so that $F$ is locally Lipschitz continuous; if $\rho^{2, i, j}>0$, the bound follows from (60) and (A.1). Therefore, on $[0, \rho]$, the drift $F\left(\zeta_{t}^{i}-\zeta_{t}^{j}\right)$ coincides with some functional $G\left(\left(\nu_{s}^{i}-\nu_{s}^{j}\right)_{0 \leq s \leq t}\right)$ of the whole path $\left(\nu_{s}^{i}-\nu_{s}^{j}\right)_{0 \leq s \leq t}, G$ being bounded and locally Lipschitz continuous w.r.t. the $L^{\infty}$-norm. We then write

$$
d \nu_{t}^{i}=\frac{1}{N} \sum_{j \neq i} G\left(\left(\nu_{s}^{i}-\nu_{s}^{j}\right)_{0 \leq s \leq t}\right) d t+\sum_{k=1}^{+\infty} \sigma_{k}\left(\chi_{t}^{i}\right) d W_{t}^{k}, \quad i \in\{1, \ldots, d\}
$$

for $t \in[0, \rho]$, with $\rho=\inf _{i \neq j} \rho^{i, j}$. (Obviously, (62) is also satisfied by $\zeta^{\prime}$.) Eq. (62) reads as a functional equation driven by bounded and locally Lipschitzcontinuous coefficients, the Lipschitz constants of the coefficients on any balls 
being finite random variables depending upon $\omega$ through the initial condition $Z_{0}$ only. Lemma 29 follows from $\mathbb{P}\left\{\sup _{0 \leq s \leq \rho}\left|\nu_{s}-\nu_{s}^{\prime}\right|=0 \mid Z_{0}\right\}=1$.

Step 4. We have proven weak existence and strong uniqueness for a.e. initial condition $z \in \Gamma_{N}$. Following the proof by Yamada and Watanabe in the finite-dimensional case, we deduce that both strong existence and strong uniqueness hold for a.e. initial condition $z \in \Gamma_{N}$.

\subsection{No coalescence for any initial condition in $\Gamma_{N}$}

We now establish the main result of the paper. To do so, we first prove

Lemma 30 For any $z \in \Gamma_{N}$, there exists a unique solution $\left(\varphi_{t}(z)\right)_{0 \leq t \leq \tau_{z}}$ to (13), with $z$ as initial condition, on the interval $\left[0, \tau_{z}\right]$, where $\tau_{z}=\inf \{t \geq$ $\left.0: \varphi_{t}(z) \in \Gamma_{N}^{\complement}\right\}$. Moreover, the mapping $\Gamma_{N} \ni z \mapsto\left(\varphi_{t \wedge \tau_{z}}(z)\right)_{t \geq 0} \in$ $\mathcal{C}\left([0,+\infty), \mathbb{R}^{2 N d}\right)$ is measurable.

Proof. The whole difficulty is to handle the possible coalescence of the particles in the space of positions. By induction, we build a non-decreasing sequence of stopping times $\left(\tau_{z}^{n}\right)_{n \in \mathbb{N}}$ such that $\tau_{z}^{n} \rightarrow \tau_{z}$ a.s. as $n$ tends to $+\infty$ and (13) has a unique solution $\left(\varphi_{t}(z)\right)_{0 \leq t \leq \tau_{z}^{n}}$ on each $\left[0, \tau_{z}^{n}\right]$ with $z$ as initial solution for any $n \in \mathbb{N}$. The stopping time $\tau_{0}$ is set equal to 0 . Given $\left(\varphi_{t}(z)=\left(\chi_{t}(z), \nu_{t}(z)\right)\right)_{0 \leq t \leq \tau_{z}^{n}}$, for some $n \in \mathbb{N}$, we can follow the proof of Lemma 29 and build a (unique) solution $\left(\varphi_{t}(z)\right)_{\tau_{z}^{n} \leq t \leq \tau_{z}^{n+1}}$ to (13) on $\left[\tau_{z}^{n}, \tau_{z}^{n+1}\right]$, where $\tau_{z}^{n+1}=\tau_{z} \wedge \tau_{z}^{\prime, n+1}$, where $\tau_{z}^{\prime, n+1}=\inf _{i \neq j} \rho^{i, j, n+1}$, with $\rho^{i, j, n+1}=\max \left(\rho^{1, i, j, n+1}, \rho^{2, i, j, n+1}\right)$,

$$
\begin{aligned}
& \rho^{1, i, j, n+1}=\inf \left\{t \geq \tau_{z}^{n}:\left|\chi_{t}^{i}(z)-\chi_{t}^{j}(z)\right| \leq\left|\chi_{\tau_{z}^{n}}^{i}(z)-\chi_{\tau_{z}^{n}}^{j}(z)\right| / 2\right\}, \\
& \rho^{2, i, j, n+1}=\inf \left\{t>\tau_{z}^{n}:\left|\chi_{t}^{i}(z)-\chi_{t}^{j}(z)\right| \leq\left(t-\tau_{z}^{n}\right)\left|\nu_{\tau_{z}^{n}}^{i}(z)-\nu_{\tau_{z}^{n}}^{j}(z)\right| / 2\right\} .
\end{aligned}
$$

Clearly, the sequence $\left(\tau_{z}^{n}\right)_{n \in \mathbb{N}}$ is non-decreasing.

On each step, existence and uniqueness hold since Eq. (13) can be written as a functional SDE on the interval $\left[\tau_{z}^{n}, \tau_{z}^{n+1}\right]$ with bounded and locally Lipschitz-continuous coefficients. (As already emphasized in the proof of Lemma 29, the Lipschitz constants of the coefficients on bounded sets depend on the initial position $\varphi_{\tau_{z}^{n}}(z)$. Anyhow, this has no consequences.)

Almost-surely, the sequence $\left(\tau_{z}^{n}\right)_{n \in \mathbb{N}}$ cannot have an accumulation point before $\varphi(z)$ hits $\Gamma_{N}^{\complement}$, as otherwise there would be a blow-up for the modulus of continuity of $\varphi(z)$. Again, the precise argument goes back to the proof of Lemma 29: the length $\tau_{z}^{n+1}-\tau_{z}^{n}$ depends on the modulus of continuity of 
the path $\left(\varphi_{t}(z)\right)_{\tau_{z}^{n} \leq t \leq \tau_{z}^{n+1}}$-the length of the interval is controlled from below when the modulus is controlled from above-, on the $\operatorname{distance} \operatorname{dist}\left(\varphi_{\tau_{z}^{n}}(z), \Gamma_{N}^{\complement}\right)$ -the length of the interval is controlled from below when the distance is away from zero- and on the norm $\left|\varphi_{\tau_{z}^{n}}(z)\right|$-the length of the interval is controlled from below when the norm is away from $+\infty-$. The modulus of continuity is controlled in terms of the bounds of the coefficients by Kolmogorov's criterion, the norm of $\varphi(z)$ is controlled in terms of the bounds of the coefficients as well, and the distance from $\varphi(z)$ to $\Gamma_{N}^{\complement}$ is bounded from below on any $\left[0, \tilde{\tau}_{z}^{\epsilon}\right]$, with $\tilde{\tau}_{z}^{\epsilon}=\inf \left\{t \geq 0: \operatorname{dist}\left(\varphi_{t}(z), \Gamma_{N}^{\complement}\right) \leq \epsilon\right\}$, for $\epsilon>0$. This proves that, a.s., $\sup _{n \geq 1} \tau_{z}^{n} \geq \tilde{\tau}_{z}^{\epsilon}$ for any $\epsilon>0$, that is $\sup _{n \geq 1} \tau_{z}^{n} \geq \tilde{\tau}_{z}^{\epsilon}=\tau_{z}$.

Proof. (Theorem 22.) We now complete the proof. We add a point $\Delta$ to $\mathbb{R}^{2 N d}$ and set $\varphi_{t}(z)=\Delta$ for $t \geq \tau_{z}, z \in \Gamma_{N}$, when $\tau_{z}<\infty$. The resulting family of processes $\left(\varphi_{t}(z)\right)_{t \geq 0}, z \in \Gamma_{N}$, has $\Gamma_{N} \cup \Delta$ as state space. It is a homogeneous Markov process. By Theorem 28, $\mathbb{P}\left\{\tau_{z}=+\infty\right\}=1$ for a.e. $z \in \mathbb{R}^{2 N d}$. In particular, we can write, for any $0<\varepsilon<T$,

$$
\mathbb{P}\left\{\varphi_{[\varepsilon, T]}(z) \in \Gamma_{N}\right\}=\int_{\Gamma_{N} \cup\{\Delta\}} \mathbb{P}\left\{\varphi_{[0, T-\varepsilon]}\left(z^{\prime}\right) \in \Gamma_{N}\right\} \mu_{\varphi_{\varepsilon}(z)}\left(d z^{\prime}\right)
$$

where $\left\{\varphi_{[\varepsilon, T]}(z) \in \Gamma_{N}\right\}=\left\{\omega \in \Omega: \varphi_{t}(z)(\omega) \in \Gamma_{N}\right.$, for any $\left.t \in[\varepsilon, T]\right\}$ and $\mu_{\varphi_{\varepsilon}(z)}$ is the law of $\varphi_{\varepsilon}(z)$. By Theorem 28, we can find a Borel subset $\mathcal{N} \subset \mathbb{R}^{2 N d}$ of zero Lebesgue measure such that $\mathbb{P}\left\{\varphi_{[0, T-\varepsilon]}(z) \in \Gamma_{N}\right\}=1$ for all $z \in \mathcal{N}^{\complement}$. Then

$$
\begin{aligned}
& \mathbb{P}\left\{\varphi_{[\varepsilon, T]}(z) \in \Gamma_{N}\right\} \geq \int_{\mathcal{N}^{C}} \mathbb{P}\left\{\varphi_{[0, T-\varepsilon]}\left(z^{\prime}\right) \in \Gamma_{N}\right\} \mu_{\varphi_{\varepsilon}(z)}\left(d z^{\prime}\right) \\
& =1-\mu_{\varphi_{\varepsilon}(z)}(\mathcal{N}) .
\end{aligned}
$$

Now, assume that $z=(x, v)$ is such that $x \in \Gamma_{x, N}$ (so that $z \in \Gamma_{N}$ ). Then, there exists $\varepsilon^{*}>0$ such that $\inf _{i \neq j}\left|x^{i}-x^{j}\right|>\varepsilon^{*}$. Defining $\tau_{z}^{*}=\inf \{t \geq 0$ : $\left.\inf _{i \neq j}\left|\pi_{i, x}\left(\varphi_{t}(z)\right)-\pi_{j, x}\left(\varphi_{t}(z)\right)\right| \leq \varepsilon^{*}\right\}$, we have

$$
\begin{aligned}
\mu_{\varphi_{\varepsilon}(z)}(\mathcal{N}) & \leq \mathbb{P}\left\{\varphi_{\varepsilon}^{*}(z) \in \mathcal{N}, \tau_{z}^{*}>\varepsilon\right\}+\mathbb{P}\left\{\tau_{z}^{*} \leq \varepsilon\right\} \\
& \leq \mathbb{P}\left\{\varphi_{\varepsilon}^{*}(z) \in \mathcal{N}\right\}+\mathbb{P}\left\{\tau_{z}^{*} \leq \varepsilon\right\}
\end{aligned}
$$

where $\left(\varphi_{t}^{*}(z)\right)_{t \geq 0}$ stands for the solution to (13) when driven by a Lipschitz drift that coincides with the original one on $\left\{\left(x^{1}, \ldots, x^{N}\right) \in \mathbb{R}^{2 N d}: \inf _{i \neq j} \mid x^{i}-\right.$ $\left.x^{j} \mid>\varepsilon^{*}\right\}$. Since $\mathcal{N}$ is Lebesgue-negligible and the law of $\varphi_{\varepsilon}^{*}(z)$ on $\mathbb{R}^{2 N d}$ is absolutely continuous w.r.t. Lebesgue measure when $x \in \Gamma_{x, N}$, we deduce 
that $\mathbb{P}\left\{\varphi_{\varepsilon}^{*}(z) \in \mathcal{N}\right\}=0$. (See Proposition 21.) Letting $\varepsilon$ tend to zero, we deduce that $\mathbb{P}\left\{\varphi_{[0, T]} \in \Gamma_{N}\right\}=1$. Indeed, $\cap_{\varepsilon>0}\left\{\varphi_{[\varepsilon, T]}(z) \in \Gamma_{N}\right\}=\left\{\varphi_{(0, T]}(z) \in\right.$ $\left.\Gamma_{N}\right\}=\left\{\varphi_{[0, T]}(z) \in \Gamma_{N}\right\}$ since $\varphi_{0}(z) \in \Gamma_{N}^{\complement}$ implies $\varphi_{t}(z)=\Delta$ for any $t>0$.

Assume now that $z \in \Gamma_{N}$ but $x \notin \Gamma_{x, N}$. Following the proof of Lemma 29 , we know that, $\mathbb{P}$ almost-surely, there exists a non-empty interval $(0, \rho(\omega))$ such that $\varphi_{t}(z) \in \Gamma_{x, N}, \rho$ standing for a stopping-time. (When $x^{i}=x^{j}$, $\left|\pi_{i, x}\left(\varphi_{t}(z)\right)-\pi_{j, x}\left(\varphi_{t}(z)\right)\right| \geq(t / 2)\left|v^{i}-v^{j}\right|$ for $t>0$ small and is thus non-zero for $t>0$ small.) In particular, $\tau_{z}(\omega) \geq \rho(\omega)$. For any $\delta \in(0, \varepsilon)$, we have

$$
\begin{aligned}
\mu_{\varphi_{\varepsilon}(z)}(\mathcal{N}) & \leq \mathbb{P}\left\{\varphi_{\varepsilon}(z) \in \mathcal{N}, \delta<\rho\right\}+\mathbb{P}\{\rho \leq \delta\} \\
& \leq \mathbb{P}\left\{\varphi_{\varepsilon}(z) \in \mathcal{N}, \Pi_{x}\left(\varphi_{\delta}(z)\right) \in \Gamma_{x, N}\right\}+\mathbb{P}\{\rho \leq \delta\} .
\end{aligned}
$$

By Markov property, we then claim

$$
\begin{aligned}
& \mathbb{P}\left\{\varphi_{\varepsilon}(z) \in \mathcal{N}, \Pi_{x}\left(\varphi_{\delta}(z)\right) \in \Gamma_{x, N}\right\} \\
& =\int_{\mathbb{R}^{2 N d}} \mathbb{P}\left\{\varphi_{\varepsilon-\delta}\left(z^{\prime}\right) \in \mathcal{N}\right\} \mathbf{1}_{\left\{\Pi_{x}\left(z^{\prime}\right) \in \Gamma_{x, N}\right\}} \mu_{\varphi_{\delta}(z)}\left(d z^{\prime}\right) .
\end{aligned}
$$

Going back to (63), we write $\varepsilon$ as $\varepsilon=\varepsilon_{1}+\varepsilon_{2}$, with $\varepsilon_{1}, \varepsilon_{2}>0$. Choosing $\delta=\varepsilon_{1}$ in (65), we have $\varepsilon-\delta=\varepsilon_{2}$ in (66). By (64) and by Lebesgue's dominated convergence Theorem, we know that the right-hand side in (66) tends to 0 as $\varepsilon_{2}=\varepsilon-\delta$ tends to 0 . Passing to the limit in (63) and using (65), this says that $\mathbb{P}\left\{\varphi_{\left[\varepsilon_{1}, T\right]}(z) \in \Gamma_{N}\right\} \geq 1-\mathbb{P}\left\{\rho \leq \varepsilon_{1}\right\}$, for any $\varepsilon_{1}>0$. Letting $\varepsilon_{1}$ tend to zero, we obtain $\mathbb{P}\left\{\varphi_{[0, T]}(z) \in \Gamma_{N}\right\}=1$.

\section{References}

[1] L. Ambrosio, Transport equation and Cauchy problem for BV vector fields, Inventiones Math. 158 (2004), no. 2, 227-260.

[2] S. Attanasio, F. Flandoli, Zero-noise solutions of linear transport equations without uniqueness: an example, C. R. Acad. Sci. Paris, Ser. I 347 (2009) 753-756.

[3] R. Bafico, P. Baldi. Small Random Perturbations of Peano Phenomena, Stochastics 6 (1982), 279-292.

[4] J. Bec, M. Cencini, R. Hillerbrand, Heavy particles in incompressible flows: the large Stokes number asymptotics, Physica D 226 (2007), 1122 . 
[5] L. Carmack, G. Majda, Concentrations in the one-dimensional VlasovPoisson equations: additional regularizations, Phys. D 121 (1998), no. $1-2,127-162$.

[6] A. Celani, S. Rubenthaler, D. Vincenzi, Dispersion and collapse in stochastic velocity fields on a cylinder, J. Stat. Phys. 138 (2010), no. $4-5,579-597$

[7] J.-M. Delort, Existence de nappes de tourbillon en dimension deux (French), J. Amer. Math. Soc. 4 (1991), no. 3, 553-586.

[8] R.-J. DiPerna, P.-L. Lions, Ordinary differential equations, transport theory and Sobolev spaces, Inventiones Math. 98 (1989), no. 3, 511547.

[9] W. E, E. Vanden-Eijnden, A note on generalized flows, Physica D 183 (2003), 159174

[10] G. Eyink, J, Xin, Existence and uniqueness of $L^{2}$-solutions at zerodiffusivity in the Kraichnan model of a passive scalar, ...

[11] G. Falkovich, K. Gawedzki, M. Vergassola, Particles and fields in turbulence, Rev. Mod. Phys. 73 (2001), 913-975.

[12] F. Flandoli, Random Perturbations of PDEs and Fluid Dynamics Models, Ecole d'été de probabilités de Saint-Flour, Lecture Notes in Math. 2015, Springer, Berlin, 2011.

[13] F. Flandoli, M. Gubinelli, E. Priola, Well posedness of the transport equation by stochastic perturbation, Invent. Math. 180 (2010), 1-53.

[14] F. Flandoli, M. Gubinelli, E. Priola, Full well-posedness of point vortex dynamics corresponding to stochastic 2D Euler equations, arXiv:1004.1407, accepted on Stoch. Proc. Appl.

[15] M. Gradinaru, S. Herrmann, B. Roynette, A singular large deviations phenomenon, Ann. Inst. H. Poincaré Probab. Statist. 37 (2001), no. 5, $555-580$.

[16] M. Hauray, P.-E. Jabin, N-particles approximation of the Vlasov equations with singular potential, Arch. Ration. Mech. Anal. 183 (2007), 489-524. 
[17] V. Hakulinen, Passive advection and the degenerate elliptic operators $M_{n}$, Comm. Math. Phys. 235 (2003), 1-45.

[18] P. Kotelenez, Stochastic Ordinary and Stochastic Partial Differential Equations: Transition from Microscopic to Macroscopic Equations, Stochastic modelling and applied probability 58, Springer, 2008.

[19] N. V. Krylov, M. Röckner, Strong solutions of stochastic equations with singular time dependent drift, Probab. Theory Related Fields 131 (2005), 154-196.

[20] H. Kunita, Stochastic differential equations and stochastic flows of diffeomorphisms, Ecole d'été de probabilités de Saint-Flour, XL-2010, Lecture Notes in Math. 2015, Springer, Berlin, 2011.

[21] H. Kunita, Stochastic Flows and Stochastic Differential Equations, Cambridge Studies in Advanced Mathematics, vol. 24. Cambridge Univ. Press, Cambridge (1990).

[22] Y. Le Jan, On isotropic Brownian motions, Z. Wahrsch. Verw. Gebiete 70 (1985), no. 4, 609-620.

[23] Y. Le Jan, O. Raimond, Integration of Brownian vector fields, Ann. Probab. 30 (2002), no. 2, 826-873.

[24] P.-L. Lions, B. Perthame, Propagation of moments and regularity for the 3-dimensional Vlasov-Poisson system, Invent. Math. 105 (1991), 415430 .

[25] S. V. Lototskii, B. L. Rozovskii, The passive scalar equation in a turbulent incompressible Gaussian velocity field. (Russian) Uspekhi Mat. Nauk 59 (2004), no. 2(356), 105-120; translation in Russian Math. Surveys 59 (2004), no. 2, 297-312.

[26] A. J. Majda, A. L. Bertozzi, Vorticity and incompressible flow, Cambridge Texts in Applied Mathematics, 27, Cambridge University Press, Cambridge, 2002.

[27] A. J. Majda, G. Majda, Y. Zheng, Concentrations in the onedimensional Vlasov-Poisson equations, I. Temporal development and non-unique weak solutions in the single component case. Phys. D 74 (1994), no. 3-4, 268-300. 
[28] C. Marchioro, M. Pulvirenti, Mathematical Theory of Incompressible Nonviscous Fluids, Applied Mathematical Sciences, 96. Springer-Verlag, New York, 1994.

[29] A.S. Monin, A.M. Yaglom, Statistical Fluid mechanics (Vol. 2), Cambridge MIT Press, 1975.

[30] M.D. Nguyen, D. Nualart, M. Sanz, Application of Malliavin Calculus to a Class of Stochastic Differential Equations, Probab. Theory Related Fields 84 (1990), no. 4, 549-571.

[31] D. Nualart, The Malliavin Calculus and Related Topics, Springer, 1995.

[32] F. Poupaud, Diagonal defect measures, adhesion dynamics and Euler equation, Methods Appl. Anal. 9 (2002), no. 4, 533-561.

[33] A.M. Yaglom, Some Classes of Random Fields in n-Dimensional Space, Related to Stationary Random Processes, Theory Probab. Appl. 2 (1957), 273-320.

[34] Y. Zheng, A. J. Majda, Existence of global weak solutions to onecomponent Vlasov-Poisson and Fokker-Planck-Poisson systems in one space dimension with measures as initial data, Comm. Pure Appl. Math. 47 (1994), no. 10, 1365-1401.

François Delarue, Dario Vincenzi

Laboratoire J.-A. Dieudonné

UMR CNRS 7351

Université de Nice Sophia-Antipolis

Parc Valrose

06108 Cedex 02, Nice, France

delarue@unice.fr, vincenzi@unice.fr
Franco Flandoli

Dipartimento Matematica

Applicata "U. Dini"

Università di Pisa

Via Buonarroti 1

C.A.P. 56127, Pisa, Italy

flandoli@dma.unipi.it 